\title{
Alteraciones de la motilidad esofágica en la manometría de alta resolución: Clasificación de Chicago versión $4.0^{\odot}$
}

\section{Esophageal motility disorders on high-resolution manometry: Chicago classification version $4.0^{\circ}$}

\begin{abstract}
Rena Yadlapati ${ }^{1}$, Peter J. Kahrilas ${ }^{2}$, Mark R. Fox ${ }^{3,4}$, Albert J. Bredenoord ${ }^{5}$, C. Prakash Gyawali ${ }^{6}$, Sabine Roman ${ }^{7,8}$, Arash Babaei ${ }^{9}$, Ravinder K. Mittal ${ }^{1,10}$, Nathalie Rommel ${ }^{11}$, Edoardo Savarino ${ }^{12}$, Daniel Sifrim ${ }^{13}$, André Smout ${ }^{5}$, Michael F. Vaezi $i^{14}$, Frank Zerbib ${ }^{15}$, Junichi Akiyama ${ }^{16}$, Shobna Bhatia ${ }^{17}$, Serhat Bor ${ }^{18}$, Dustin A. Carlson ${ }^{2}$, Joan W. Chen ${ }^{19}$, Daniel Cisternas ${ }^{20}$, Charles Cock ${ }^{21}$, Enrique Coss-Adame ${ }^{22}$, Nicola de Bortoli ${ }^{23}$, Claudia Defilippi ${ }^{24}$, Ronnie Fass ${ }^{25}$, Uday C. Ghoshal ${ }^{26}$, Sutep Gonlachanvit ${ }^{27}$, Albis Hanii' ${ }^{28}$, Geoffrey S. Hebbard ${ }^{29}$, Kee Wook Jung ${ }^{30}$, Philip Katz ${ }^{31}$, David A. Katzka ${ }^{32}$, Abraham Khan ${ }^{33}$, Geoffrey Paul Kohn ${ }^{34}$, Adriana Lazarescu ${ }^{35}$, Johannes Lengliner ${ }^{36}$, Sumeet K. Mittal ${ }^{37}$, Taher Omari ${ }^{38}$, Moo In Park ${ }^{39}$, Roberto Penagini ${ }^{40}$, Daniel Pohl ${ }^{41}$, Joel E. Richter ${ }^{42}$, Jordi Serra ${ }^{43}$, Rami Sweis ${ }^{44}$, Jan Tack ${ }^{45}$, Roger P. Tatum ${ }^{46}$, Radu Tutuian ${ }^{47,48}$, Marcelo F. Vela ${ }^{49}$, Reuben K. Wong ${ }^{50}$, Justin C. Wu ${ }^{51}$, Yinglian $X_{1 a 0^{52}}$ y John E. Pandolfino ${ }^{2 *}$
\end{abstract}

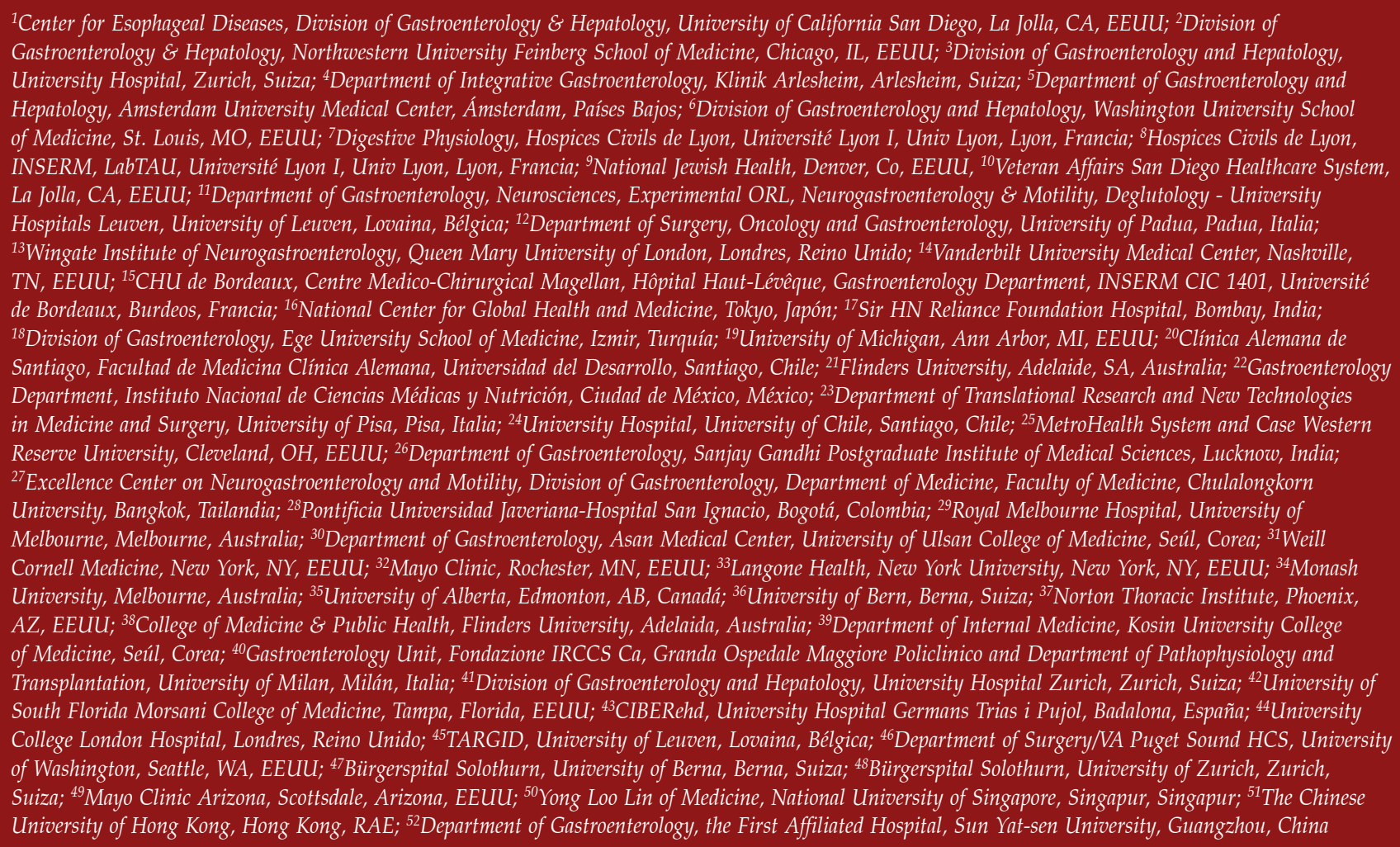

Correspondencia:

*John E. Pandolfino

Email: j-pandolfino@northwestern.edu
NeuroGastroLatam Rev. 2021;3:115-143

Recibido: 22-08-2021

Aceptado: 06-09-2021

DOI: $10.24875 /$ NGL.21000017

2462-7011/C 2021 Sociedad Latinoamericana de Neurogastroenterología, A.C. Published by Permanyer. This is an open access article under the CC BY-NC-ND license (http://creativecommons.org/licenses/by-nc-nd/4.0/). 


\section{RESUMEN}

La Clasificación de Chicago v4.0 (CCv4.0) es el esquema actualizado para clasificar las alteraciones de la motilidad esofágica utilizando la métrica de la manometría de alta resolución (HRM). Para desarrollar la CCv4.0, 52 expertos internacionales diversos separados en siete subgrupos de trabajo utilizaron una metodología formalmente validada, en un período de dos años. Las actualizaciones claves de la CCv4.0 consisten en un protocolo de HRM más riguroso y expansivo que incorpora las posiciones en decúbito supino y sedestación, así como pruebas provocadoras, una definición refinada de la obstrucción del flujo de salida de la unión esofagogástrica (EGJOO), criterios diagnósticos más estrictos para la motilidad esofágica inefectiva, y descripción de la métrica basal de la EGJ. Adicionalmente, la CCv4.0 se propuso definir el diagnóstico de alteración de la motilidad como conclusivo o no conclusivo con base en los síntomas relacionados, los hallazgos en las pruebas provocadoras, y los resultados de los estudios de apoyo como el esofagograma con tableta de bario y/o la prueba con sonda para imagen endoluminal funcional. Estos cambios buscan minimizar la ambigüedad presente en iteraciones previas de la Clasificación de Chicago; además proveen criterios más estandarizados y rigurosos para los patrones de alteración de la peristalsis y obstrucción de la EGJ.

Palabras clave: Acalasia. Espasmo esofágico. Presión de relajación integrada. Esfínter esofágico inferior. Miotomía. Endoscopia peroral.

\section{ABSTRACT}

The Chicago Classification v4.0 (CCv4.0) is the updated scheme for classifying esophageal motility disturbances using high-resolution manometry (HRM) metrics. To develop CCv4.0, fifty-two diverse international experts separated into seven sub-working groups used a formally validated methodology, over a two-year period. The key updates to CCv4.0 consist of a more rigorous and expansive HRM protocol that incorporates supine and sitting positions, as well as provocative testing; a refined definition of esophagogastric junction outflow obstruction (EGJOO), stricter diagnostic criteria for ineffective esophageal motility, and description of baseline EGJ metrics. Additionally, the CCV4.0 set out to define the diagnosis of motility impairment as conclusive or inconclusive based on the associated symptoms, the findings in provocative tests, and the results of supportive tests such as the barium tablet esophagram, and/or functional endoluminal imaging probe test. These changes seek to minimize the ambiguity present in previous iterations of the Chicago Classification; they also provide more standardized and rigorous criteria for altered patterns of peristalsis and obstruction of the EGJ.

Keywords: Achalasia. Esophageal spasm Integrated relaxation pressure. Lower esophageal sphincter. Myotomy. Peroral endoscopy.

\section{INTRODUCCIÓN}

El presente es una traducción literal al español del artículo original de Chicago 4.0 publicada en la revista Neurogastroenterology and Motility, autorizada por Wiley ${ }^{1}$. La Clasificación de Chicago categoriza las alteraciones de la motilidad esofágica por medio de un esquema algorítmico, utilizando la métrica de la manometría esofágica de alta resolución (HRM). La primera versión completa de la Clasificación de Chicago publicada en 2009 ha tenido dos actualizaciones, siendo 3.0 la versión más reciente, publicada en 2015-4. En los últimos cinco años se han expandido tanto las investigaciones como las aplicaciones clínicas de la HRM y se han introducido nuevas métricas para la prueba. Por esta razón, un Grupo Internacional de Trabajo en HRM de 52 miembros se ocupó durante dos años en desarrollar la Clasificación de Chicago versión 4.0 (CCv4.0). El presente documento hará un resumen general de la iniciativa CCv4.0 y presentará la versión actualizada de la Clasificación de Chicago sobre las alteraciones de 
la motilidad esofágica. Aunque la Clasificación de Chicago constituye un marco para clasificar las alteraciones de la motilidad esofágica con base en la $H R M$, el diagnóstico clínico final puede requerir estudios de apoyo para refinar las decisiones clínicas, particularmente en casos limítrofes o en instancias con hallazgos discordantes entre las diferentes posiciones o durante pruebas provocadoras.

\section{MÉTODOS}

El Grupo de Trabajo CCv4.0 está compuesto por 52 miembros representantes de 20 países, seleccionados por seis sociedades internacionales de motilidad. La Tabla S1 detalla las características del grupo de trabajo. La iniciativa CCv4.0 tomó un proceso de dos años (noviembre de 2018 a octubre de 2020), que incluyó tres reuniones internacionales (mayo de 2019 en San Diego, California; octubre de 2019 en Barcelona, España; y agosto de 2020, conferencia vía web), múltiples reuniones de subgrupos, y siete encuestas.

La encuesta inicial llevada a cabo en enero de 2019 identificó en la versión 3.0 las áreas prioritarias de ser actualizadas y modificadas (Tabla S1). En consecuencia, se asignaron los miembros del grupo a siete subgrupos: Protocolo de HRM estándar, Acalasia, Obstrucción del flujo de salida de la unión esofagogástrica (EGJOO), Espasmo esofágico distal (DES), Esófago hipercontráctil, Motilidad esofágica inefectiva (IEM), y Métrica de la EGJ. En cada subgrupo, dos codirectores lideraron el trabajo y un miembro no votante revisó de forma independiente la literatura de apoyo y evaluó el nivel de evidencia. A los codirectores y los miembros del subgrupo se les encargó desarrollar conceptos para definir un diagnóstico conclusivo de la alteración de la motilidad asignada a su subgrupo. También se les pidió describir escenarios no conclusivos de las alteraciones de la motilidad y el valor de los estudios de apoyo. Estos conceptos se basaron en la revisión de la literatura y consenso de expertos.

Además del consenso de expertos, la CCv4.0 empleó metodologías formalmente validadas para determinar lo apropiado de los conceptos y el nivel de evidencia para cada uno de estos conceptos. Se utilizó el Método RAND de Uso Apropiado (RAM) para determinar lo apropiado de cada concepto. Se hicieron dos rondas de votación electrónica independientes, utilizando el sistema de la Universidad de California en San Diego Research Electronic Database Capture (REDCap). Los conceptos que alcanzaron un nivel de acuerdo $\geq 80 \%$ se consideraron apropiados y se incluyeron en el CCv4.0 final ${ }^{5,6}$. Los conceptos que alcanzaron un nivel de acuerdo $\geq 85 \%$ se consideraron recomendaciones fuertes, mientras que los que alcanzaron un nivel de acuerdo del 80 a $85 \%$ se consideraron recomendaciones condicionales. Los conceptos que estuvieron cerca de alcanzar los criterios anteriores y/o aquellos que generaron controversia se discutieron en reuniones del grupo de trabajo. Adicionalmente, los conceptos que llenaron los criterios de inclusión en el CCv4.0 final se evaluaron de forma independiente para determinar su nivel de evidencia como apoyo. Para este fin se utilizó el proceso GRADE (por su acrónimo en inglés Grading of Recommendations Assessment, Development and Evaluation) cuando era posible ${ }^{7}$. Dos expertos externos al trabajo de los subgrupos, uno de ellos con entrenamiento formal en el método 
$G R A D E$, evaluaron de forma independiente la literatura de apoyo suministrada por los subgrupos. Algunos conceptos no eran adaptables al proceso GRADE por su estructura o por falta de evidencia disponible.

El presente documento resume las recomendaciones finales del grupo de trabajo CCv4.0. En revisiones técnicas separadas, específicas para cada grupo de trabajo, se resumirá el proceso de desarrollo del concepto, los resultados de la encuesta, y la literatura de apoyo. Éstas serán publicadas de forma separada en ediciones futuras de Neurogastroenterology and Motility durante los meses siguientes.

\section{PROTOCOLO Y UMBRALES DE LA HRM ESTÁNDAR}

\section{Protocolo para la HRM estándar}

Una prioridad de la Clasificación de Chicago fue estandarizar el protocolo de manometría para los laboratorios de motilidad alrededor del mundo, con el fin de facilitar la consistencia en el procedimiento, mejorar la confiabilidad diagnóstica, y promover la investigación colaborativa. El protocolo final para HRM esofágica estándar está resumido en la Figura 1 y representado en la Figura 2. La revisión técnica subsiguiente del protocolo para HRM describe los detalles de posicionamiento, maniobras, y consistencia del bolo.

Antes del procedimiento los pacientes deben completar al menos cuatro horas de ayuno (se permiten pequeñas cantidades de líquidos claros) y se debe obtener consentimiento informado.
El estudio comienza en posición de decúbito supino (Figura 2A). Después de posicionar el catéter, se da un período de reposo de mínimo 60 segundos para permitir la adaptación y a continuación se confirma la posición del catéter utilizando un mínimo de tres inspiraciones profundas. Enseguida se captura una medición de línea de base durante al menos 30 segundos para permitir la identificación de las referencias anatómicas incluyendo el esfínter esofágico superior (UES), el esfínter esofágico inferior (LES), el punto de inversión respiratoria $(R I P)$ y la presión basal de la EGJ. Posteriormente se dan 10 tragos de agua o solución salina (cuando se utiliza manometría de alta resolución con impedanciometría) a temperatura ambiente. Se deben esperar al menos 30 segundos entre las degluciones para evitar efectos de inhibición deglutoria. Finalmente, se administra una secuencia de degluciones múltiples repetitivas $(M R S)$, consistente en cinco tragos de $2 \mathrm{ml}$ administrados con una jeringa de $10 \mathrm{ml}$ a intervalos de 2-3 segundos. La secuencia MRS se puede repetir hasta tres veces en caso de intento fallido o de respuesta contráctil anormal ${ }^{8,9}$.

A continuación, se cambia la posición del paciente de decúbito a sedestación a $80^{\circ}$ o más, con las piernas colgando fuera del borde de la cama, pero sin encorvarse ni inclinarse hacia adelante (Figura 2B). Luego del cambio de posición, nuevamente se esperan al menos 60 segundos para permitir la adaptación, se valora la posición del catéter con un mínimo de tres inspiraciones profundas, y se toma un registro basal de al menos 30 segundos para permitir la identificación de las referencias anatómicas. Luego se dan al menos cinco tragos de $5 \mathrm{ml}$. Se deben esperar al menos 30 segundos entre tragos para evitar efectos de inhibición deglutoria. Finalmente, se da un 
Protocolo estándar de manometría esofágica de alta resolución. Clasificación de Chicago versión 4.0

Procedimiento para realizar el estudio

Empiece el estudio en posición supina (utilice valores normativos para la posición supina)

- Espere la adaptación por un período $\geq 60 \mathrm{~s}$

- Documente la posición con al menos tres inspiraciones profundas

- Haga un registro basal $\geq 30 \mathrm{~s}$

- Administre 10 tragos húmedos (5 ml) en supino

- Administre una secuencia de múltiples degluciones rápidas (MRS) (pueden repetirse hasta tres secuencias de MRS en caso de intento fallido o respuesta anormal)

Cambie la posición a sedestación (utilice valores normativos para la posición sedente)

- Espere la adaptación por un período $\geq 60 \mathrm{~s}$

- Documente la posición con al menos tres inspiraciones profundas

- Haga un registro basal $\geq 30 \mathrm{~s}$

- Administre $\geq 5$ tragos húmedos $(5 \mathrm{ml})$ en sedestación

- Administre un reto de bebida rápida

Si no encuentra un trastorno mayor de la motilidad, considere las siguientes evaluaciones manométricas:

- En caso de alta probabilidad de obstrucción del flujo de salida no detectada: prueba de deglución de sólidos, prueba de alimento sólido, y/o provocación farmacológica

- En caso de sospecha de trastorno con rumiación/eructo: observación de impedanciometría posprandial de alta resolución

Si los hallazgos son equívocos y/o sospecha una obstrucción que no cumple criterios para acalasia, considere los siguientes estudios de apoyo:

- Esofagograma de bario cronometrado, preferiblemente con tableta

- Planimetría de imagen de luz funcional endoluminal (FLIP)

\section{Consideraciones}

Antes del procedimiento los pacientes deben ayunar al menos cuatro horas y se debe obtener consentimiento informado. El Grupo de Trabajo de la CCv4.0 recomienda usar un catéter para manometría de alta resolución de estado sólido con los sensores de impedancia combinados, con separaciones menores de $2 \mathrm{~cm}$. Sin embargo, el protocolo y la clasificación se pueden realizar con catéteres perfundidos con agua utilizando los valores normativos apropiados. El protocolo diseñado por el grupo de trabajo de la CCv4.0 se considera el protocolo óptimo, pero los profesionales pueden modificarlo con base en sus limitaciones de recursos y de tiempo siempre que se apliquen los valores normativos y que las otras posiciones y pruebas provocadoras se utilicen adecuadamente. Los profesionales que decidan iniciar el estudio en la posición sedente deben considerar la administración de 10 tragos en sedestación.

La clasificación se basa en la posición primaria en la cual se administran 10 tragos, ya sea supina o sedente. La valoración de la deglución en la posición secundaria y con la provocación suministra datos de apoyo.

Además de la métrica de la Clasificación de Chicago v3.0, el informe final debe incluir las mediciones basales de la unión esofagogástrica (EGJ) y los síntomas experimentados durante el estudio y dentro de los $15 \mathrm{~s}$ siguientes a una disfunción de la motilidad.

Figura 1. Protocolo estándar de manometría esofágica de alta resolución: Clasificación de Chicago versión 4.0. 
A

Protocolo en posición supina. Cortesía de University of California San Diego Center for Esophageal Diseases.

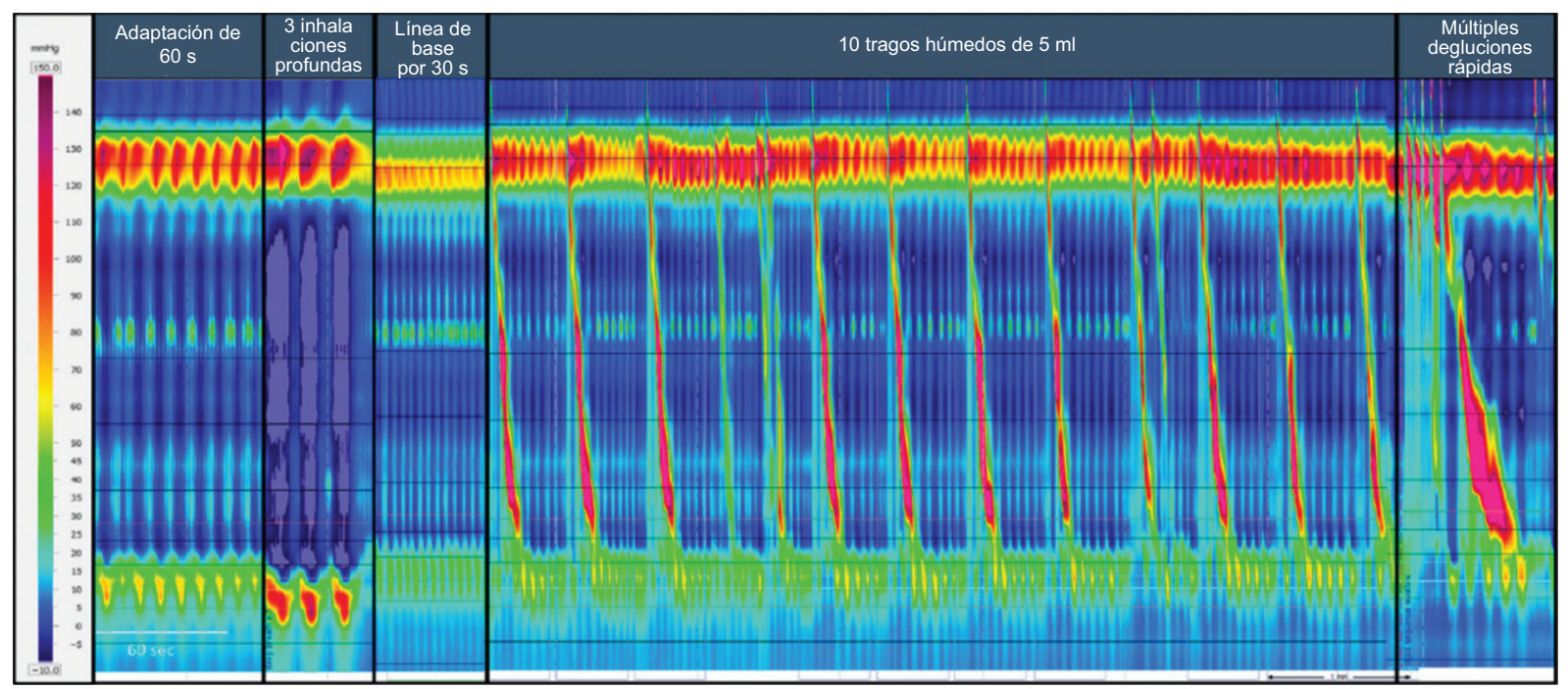

B

Protocolo en posición sedente. Cortesía de University of California San Diego Center for Esophageal Diseases.

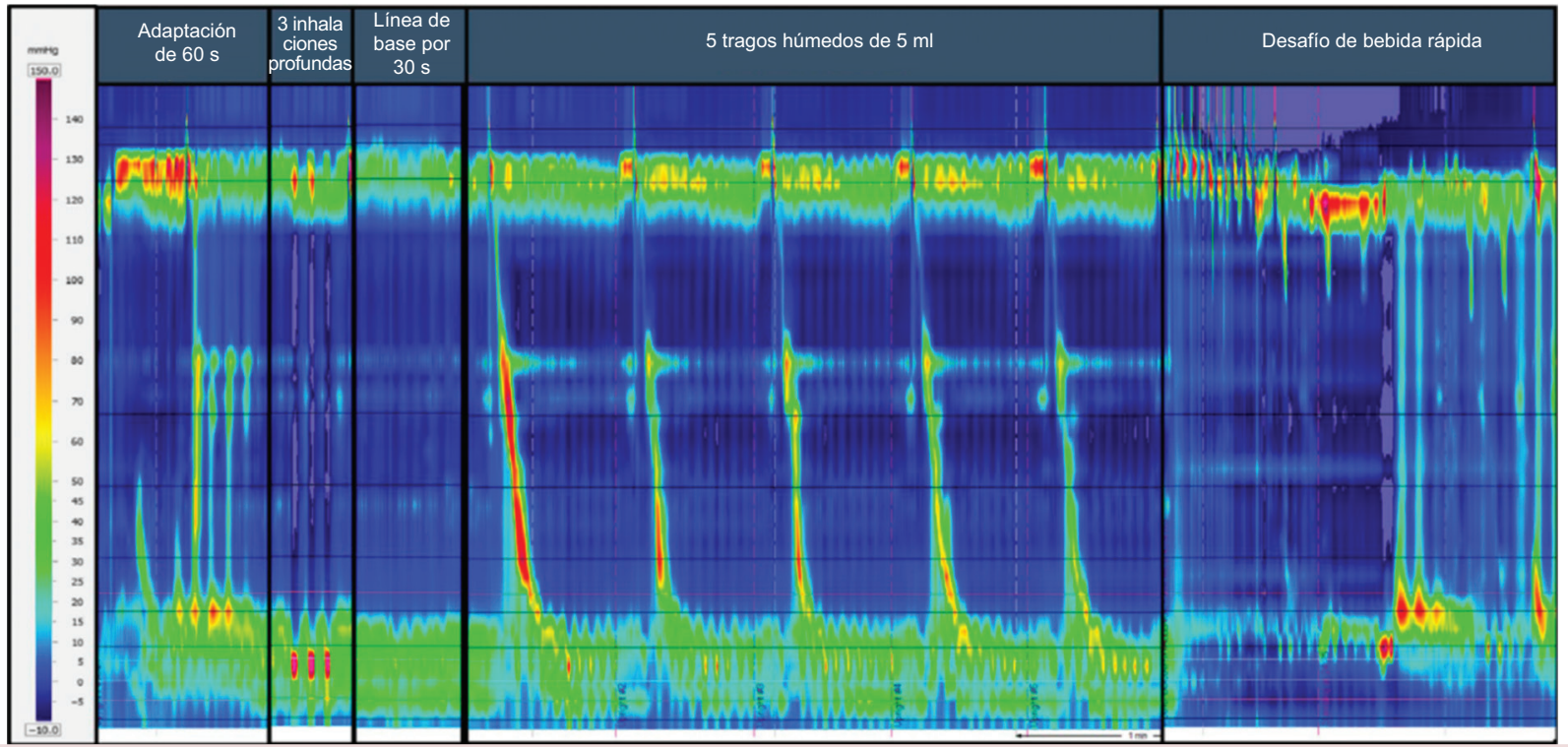

Figura 2. Imágenes de manometría de alta resolución que representan el protocolo estándar. A: la posición supina incluye un período de adaptación de $60 \mathrm{~s}$, tres inhalaciones profundas, un período basal de $30 \mathrm{~s}, 10$ tragos húmedos de $5 \mathrm{ml}$, y al menos una prueba de múltiples degluciones rápidas. B: cambio a posición sedente seguido de un período de adaptación de $60 \mathrm{~s}$, tres inhalaciones profundas, un período basal de $30 \mathrm{~s}, 5$ tragos húmedos de $5 \mathrm{ml}$, y un desafío de bebida rápida.

reto de bebida rápida $(R D C)$ con $200 \mathrm{ml}$ de agua ingeridos tan rápido como sea posible, a través de un pitillo/popote/pajilla ${ }^{10}$.

Cuando no se identifica evidencia conclusiva de una alteración mayor de la motilidad, o los resultados del protocolo no son totalmente consistentes con la presentación clínica, y/o los hallazgos no explican los síntomas del paciente, se pueden considerar medidas adicionales de apoyo de la HRM. Las medidas adicionales incluyen la prueba de 
deglución de sólidos y la prueba de comida sólida para evaluar obstrucción de la EGJ, y/o la observación posprandial de rumiación y/o trastorno con eructo ${ }^{11}$. La provocación farmacológica, si está disponible, también se puede practicar para ayudar a sustentar el diagnóstico de una verdadera alteración obstructiva de la EGJ (Figuras S1-S3) ${ }^{12}$.

Adicionalmente, frente a resultados equívocos y/o sospecha de EGJOO que no llena los criterios de acalasia, se debe obtener un esofagograma de bario seriado (TBE), preferiblemente junto con un trago de tableta de bario, y/o una prueba con sonda para imagen funcional endoluminal (FLIP). Estas pruebas independientes de apoyo permiten evaluar obstrucción de la EGJ ${ }^{13,14}$.

El Grupo de Trabajo de CCv4.0 recomienda usar un catéter para HRM de estado sólido con menos de $2 \mathrm{~cm}$ de espaciado entre los sensores. Sin embargo, el protocolo y la clasificación se pueden realizar con catéteres de perfusión con agua (con la posibilidad de tragos y maniobras limitados a la posición supina) utilizando los valores normativos apropiados. Se recomienda, aunque no es indispensable, utilizar manometría de alta resolución con impedanciometría para evaluar de manera óptima la presión intrabolo, el aclaramiento del bolo y el flujo del bolo a través de la EGJ. El protocolo debe ser ejecutado de la forma más eficiente posible para minimizar una potencial desviación de presión, reducir la incomodidad y mejorar la tolerancia del paciente.

\section{Umbrales y definiciones diagnósticas}

La CCv4.0 utilizó una métrica de HRM basada en: evaluación de la relajación deglutoria de LES / EGJ mediante presión integrada de relajación (IRP); medición del vigor contráctil del cuerpo esofágico mediante la integrada de la contractilidad distal (DCI); integridad del frente de onda contráctil con un contorno isobárico de $20 \mathrm{mmHg}$; y latencia de la inhibición deglutoria mediante latencia distal ( $D L)$ (Tabla 1). Los umbrales para la IRP mediana son más altos en la posición supina que en la sedente. Los umbrales para la $D C I$ y la $D L$ son los mismos para ambas posiciones.

\section{Presión InTEgRada de RELAJACIÓN}

- El umbral para la IRP mediana en la posición supina es $15 \mathrm{mmHg}$ para los sistemas Medtronic y $22 \mathrm{mmHg}$ para los sistemas Laborie/Diversatek (GRADE moderado, recomendación fuerte) ${ }^{15-18}$.

- El umbral para la IRP mediana en la posición supina es $12 \mathrm{mmHg}$ para los sistemas Medtronic y $12 \mathrm{mmHg}$ para los sistemas Laborie/Diversated (GRADE bajo, recomendación fuerte $)^{17-19}$.

\section{CONTRACTILIDAD EN EL CUERPO ESOFÁGICO}

La contractilidad en el cuerpo esofágico se valora mediante el vigor contráctil y el patrón contráctil ${ }^{16,17}$.

- Contracción normal: DCI entre 450 y 8,000 $\mathrm{mmHg} \bullet \bullet^{\bullet} \mathrm{cm}$

- Deglución inefectiva: incluye cualquiera de los siguientes:

a. Contracción débil: DCI entre 100 y 450 $\mathrm{mmHg} \bullet \mathrm{s} \bullet \mathrm{cm}$

b. Peristalsis fallida: $\mathrm{DCI}<100 \mathrm{mmHg} \bullet \mathrm{s}^{\bullet} \mathrm{cm}$

c. Deglución fragmentada: defecto de 
TABLA 1. Métrica y umbrales de la manometría de alta resolución

\begin{tabular}{|c|c|c|c|c|}
\hline Evaluación & $\begin{array}{l}\text { Métrica de la } \\
\text { topografía de } \\
\text { presión }\end{array}$ & Definición & Umbral diagnóstico & $\begin{array}{l}\text { Consideraciones } \\
\text { adicionales }\end{array}$ \\
\hline $\begin{array}{l}\text { Presión de } \\
\text { relajación a lo } \\
\text { largo de la unión } \\
\text { esofagogástrica } \\
\text { en respuesta a } \\
\text { la deglución }\end{array}$ & $\begin{array}{l}\text { Presión de } \\
\text { relajación integrada } \\
(I R P)\end{array}$ & $\begin{array}{l}\text { Promedio de los } 4 \text { s de } \\
\text { máxima relajación } \\
\text { deglutoria en la ventana de } \\
\text { 4-s comenzando en la } \\
\text { relajación del UES, contigua } \\
\text { o no, referenciada a la } \\
\text { presión gástrica }\end{array}$ & $\begin{array}{l}\text { Relajación anormal de IRP deglutoria: } \\
\text { - IRP mediana en supino } \geq 15 \mathrm{mmHg} \\
\text { (Medtronic) } \\
\text { - IRP mediana en supino } \geq 22 \mathrm{mmHg} \\
\text { (Laborie/Diversatek) } \\
\text { - IRP mediana en sedestación } \geq 12 \\
\mathrm{mmHg} \text { (Medtronic) } \\
\text { - IRP mediana en sedestación } \geq 15 \\
\mathrm{mmHg} \text { (Laborie/Diversatek) }\end{array}$ & $\begin{array}{l}\text { IRP }>12 \mathrm{mmHg} \text { (Medtron- } \\
\text { ic) en el desafío de bebida } \\
\text { rápida (RDC) } \\
\text { o IRP }>25 \mathrm{mmHg} \\
\text { (Medtronic) en la prueba } \\
\text { de alimento sólido, apoya } \\
\text { obstrucción del flujo de } \\
\text { salida }\end{array}$ \\
\hline \multirow[t]{2}{*}{$\begin{array}{l}\text { Peristalsis } \\
\text { esofágica }\end{array}$} & $\begin{array}{l}\text { Integral de } \\
\text { contractilidad distal } \\
(D C /) \text { - vigor } \\
\text { contráctil }\end{array}$ & $\begin{array}{l}\text { Contracción esofágica } \\
\text { distal que en términos de } \\
\text { amplitud } x \text { duración } x \\
\text { longitud }\left(\mathrm{mmHg} \bullet^{\bullet} \mathrm{cm}\right) \\
\text { excede } 20 \mathrm{mmHg} \text { desde la } \\
\text { zona de transición al borde } \\
\text { proximal del LES }\end{array}$ & 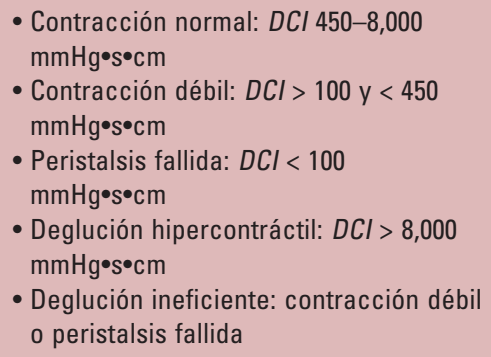 & $\begin{array}{l}\text { Respuesta contráctil intac- } \\
\text { ta con degluciones } \\
\text { múltiples rápidas (MRS): } \\
\text { Durante } M R S, \mathrm{DCl}<100 \\
\text { mmHg•s॰cm } \\
\text { y luego de } \mathrm{MRS}, \mathrm{DCl} \\
\text { mayor al } \mathrm{DCl} \text { promedio en } \\
\text { deglución única }\end{array}$ \\
\hline & $\begin{array}{l}\text { Integridad del frente } \\
\text { de onda contráctil }\end{array}$ & $\begin{array}{l}\text { Peristalsis contigua en un } \\
\text { contorno isobárico de } 20 \\
\mathrm{mmHg}\end{array}$ & $\begin{array}{l}\text { - Deglución ineficiente: interrupción } \\
\text { peristáltica }>5 \mathrm{~cm} \text { en el escenario de } \\
\text { una } \mathrm{DCl} \geq 450 \\
\mathrm{mmHg} \cdot \mathrm{s}^{\bullet} \mathrm{cm}\end{array}$ & \\
\hline $\begin{array}{l}\text { Latencia de la } \\
\text { inhibición } \\
\text { deglutoria }\end{array}$ & Latencia distal (DL) & $\begin{array}{l}\text { Intervalo entre la relajación } \\
\text { del UES y el CPD }\end{array}$ & $\begin{array}{l}\text { - Contracción prematura/espástica: } D L \\
<4.5 \mathrm{~s} \text { en el escenario de una } \mathrm{DCl} \geq 450 \\
\mathrm{mmH}{ }^{\bullet} \bullet \mathrm{cm}\end{array}$ & \\
\hline Presurización & Contorno isobárico & & $\begin{array}{l}\text { - Presurización panesofágica: contorno } \\
\text { isobárico } \geq 30 \mathrm{mmHg} \\
\text { - Presurización intrabolo: contorno } \\
\text { isobárico } \geq 20 \mathrm{mmHg} \text { en supino } \\
\text { (Medtronic) }\end{array}$ & $\begin{array}{l}\text { Apoyan obstrucción al } \\
\text { flujo de salida: presur- } \\
\text { ización panesofágica } \\
>20 \mathrm{mmHg} \text { en el RDC o la } \\
\text { prueba de alimento sólido }\end{array}$ \\
\hline
\end{tabular}

UES: esfínter esofágico superior; LES: esfínter esofágico inferior.

peristalsis $>5 \mathrm{~cm}$ en la zona de transición con un contorno isobárico de $20 \mathrm{mmHg}$ en el escenario de una $D C I$ $\geq 450 \mathrm{mmHg} \bullet \mathrm{s} \bullet \mathrm{cm}$

- Deglución hipercontráctil: DCI $>8,000 \mathrm{mmHg} \bullet \mathrm{s} \bullet \mathrm{cm}$.

a. La literatura disponible acerca de los valores normales sugiere que el umbral de $8,000 \mathrm{mmHg} \bullet \mathrm{s} \bullet \mathrm{cm}$ es adecuado para los diferentes sistemas de HRM.

\section{LATENCIA DE LA INHIBICIÓN DEGLUTORIA}

La latencia distal se mide como el intervalo desde el principio de la relajación del UES hasta el punto de la desaceleración contráctil $(C D P)$. El CDP es el punto de inflexión entre la fase rápida proximal y la fase lenta distal de la contracción esofágica, localizado en los tres centímetros proximales de la zona de alta presión de la EGJ predeglución. Fisiológicamente, este punto probablemente 
representa la transición de la contracción muscular lisa del cuerpo esofágico a la poscontracción del LES.

- Contracción prematura: latencia distal menor a 4.5 segundos en el escenario de una $D C I \geq 450 \mathrm{mmHg} \bullet \mathrm{s} \bullet \mathrm{cm}^{17}$.

Si es difícil determinar el $C D P$, se puede trazar una línea $2-3 \mathrm{~cm}$ por encima del aspecto proximal de la zona de alta presión de la $E G J$ predeglución. La $D L$ se puede determinar midiendo el tiempo desde el inicio de la relajación del UES hasta la intersección con el frente de onda contráctil. Es importante extender esta línea horizontal hasta la contracción y no hasta el frente de presurización que se puede segmentar por delante del frente de onda contráctil. La actividad contráctil esofágica se debe distinguir de otras causas de elevación de la presión en el esófago distal, como lo son la presión intrabolo o un artefacto (GRADE muy bajo, recomendación fuerte) $)^{20,21}$.

\section{PRESURIZACIÓN}

- Presurización panesofágica: el punto de corte para la presurización panesofágica cuando se utiliza la herramienta de contorno isobárico se fijó en $30 \mathrm{mmHg}$ (GRADE muy bajo, recomendación fuerte) $)^{22}$.

- Presurización intrabolo: el umbral de presión intrabolo para degluciones en supino con el sistema Medtronic es $20 \mathrm{mmHg}$ cuando se utiliza la herramienta de contorno isobárico (GRADE moderado, recomendación fuerte $)^{15,23,24}$.

\section{RESPUESTA A LA PROVOCACIÓN}

- Degluciones múltiples repetitivas: una respuesta intacta a las MRS se define como la ausencia de contractilidad del cuerpo esofágico $(D C I<100$ $\mathrm{mmHg} \bullet \mathrm{s}^{\bullet} \mathrm{cm}$ ) con inhibición deglutoria completa del LES durante las degluciones repetidas, y el aumento de las contracciones post-MRS (DCI post-MRS mayor que la $D C I$ promedio con deglución única, Tabla 2) ${ }^{8,25-27}$. La contracción post-MRS debe ser verdadera contractilidad peristáltica y no artificio o presurización.

- Reto de toma rápida $(R D C)$ : la respuesta intacta al $R D C$ se define como la ausencia de contractilidad del cuerpo esofágico $(D C I<100 \mathrm{mmHg} \bullet \mathrm{s} \bullet \mathrm{cm})$ con inhibición deglutoria completa del LES durante el $R D C$. Son criterios para obstrucción del flujo de salida la IRP $>12 \mathrm{mmHg}$ (con el sistema Medtronic) en los primeros 30 segundos del $R D C$ y la presurización panesofágica $>20 \mathrm{mmHg}$ (GRADE bajo, recomendación condicional $)^{10,27-30}$. La presencia de una secuencia de contracción normal luego del $R D C$ es un marcador específico de contractilidad normal. Sin embargo, esta secuencia no está presente en todos los controles sanos ${ }^{10,28,29}$.

- Prueba de deglución con sólidos: una respuesta contráctil intacta del cuerpo esofágico a la ingestión de sustancias sólidas requiere la presencia de degluciones faríngeas $>20 \%$ seguidas de una contracción esofágica efectiva definida por $D C I>1,000$ $\mathrm{mmHg} \bullet \mathrm{s} \bullet \mathrm{cm}$, sin una pausa prolongada 
Tabla 2. Medidas manométricas de apoyo que pueden aumentar la confianza en el diagnóstico de un trastorno

\begin{tabular}{|c|c|c|}
\hline Medida de apoyo & Protocolo & Respuesta normal \\
\hline $\begin{array}{l}\text { Múltiples degluciones rápidas } \\
\text { (MRS) }\end{array}$ & $\begin{array}{l}5 \text { degluciones de } 2 \mathrm{ml} \text { de líquido a } \\
\text { intervalos de } 2-3 \mathrm{~s}\end{array}$ & $\begin{array}{l}\text { Ausencia de contractilidad del cuerpo esofágico (DCI }<100 \\
\mathrm{mmH} \bullet^{\bullet} \mathrm{cm} \text { ) con inhibición deglutoria completa del } L E S \\
\text { durante las } M R S \text { y aumento de las contracciones post- } M R S \\
\text { (DCI post-MRS mayor que el } D C l \text { promedio en deglución única). }\end{array}$ \\
\hline Desafío de bebida rápida (RDC) & Toma rápida de $200 \mathrm{ml}$ de líquido & $\begin{array}{l}\text { Ausencia de contractilidad del cuerpo esofágico }(D C l<100 \\
\text { mmHg•s॰cm) con inhibición deglutoria completa del } L E S \\
\text { durante el } R D C \text { y sin evidencia de trastorno mayor de la } \\
\text { motilidad post- } R D C \text {. }\end{array}$ \\
\hline Prueba de deglución de sólido & $\begin{array}{l}10 \text { degluciones de } \sim 1 \mathrm{~cm} 3 \text { de sólido } \\
\text { blando (p. ej., pan, arroz cocinado } \\
\text { blando, malvavisco) }\end{array}$ & $\begin{array}{l}\text { Presencia de degluciones faríngeas }>20 \% \text { seguidas de contrac- } \\
\text { ción esofágica efectiva, definida por } \mathrm{DCl}>1,000 \mathrm{mmHg} \bullet \mathrm{s}^{\bullet} \mathrm{cm} \text { y } \sin \\
\text { interrupción prolongada }(>5 \mathrm{~cm} \text { ) en el frente contráctil. }\end{array}$ \\
\hline Prueba de alimento sólido (STM) & $\begin{array}{l}\text { Alimento sólido blando (p. ej., arroz } \\
\text { cocinado blando, pan), } 200 \mathrm{~g} \text { ingeridos al } \\
\text { ritmo normal para el paciente. El estudio } \\
\text { se detiene si la STM no se completa en } \\
8 \text { min. }\end{array}$ & $\begin{array}{l}\text { Presencia de degluciones faríngeas }>20 \% \text { seguidas de contrac- } \\
\text { ción esofágica efectiva definida por } \mathrm{DCl}>1,000 \mathrm{mmHg} \bullet \mathrm{s}^{\bullet} \mathrm{cm} \text { y sin } \\
\text { interrupción prolongada }(>5 \mathrm{~cm} \text { ) en el frente contráctil. } \\
\text { Sin síntomas durante la } S T M \text { (cualquier síntoma debe ser } \\
\text { consignado en el registro electrónico para evaluar la asociación } \\
\text { con motilidad o función anormal). La alimentación lenta con } \\
\text { ingestión }<200 \mathrm{~g} \text { en } 8 \text { min también se considera anormal. }\end{array}$ \\
\hline $\begin{array}{l}\text { Alimento posprandial (manometría } \\
\text { de alta resolución con impedan- } \\
\text { ciometría) }\end{array}$ & $\begin{array}{l}\text { Administración de STM o un alimento } \\
\text { identificado por el paciente como } \\
\text { inductor de síntomas, seguido de } \\
\text { monitoreo extendido (mínimo } 10 \text { min y } \\
\text { aparición de actividad anormal) }\end{array}$ & $\begin{array}{l}\text { Ausencia de síntomas y de motilidad o función anormal durante } \\
\text { el período posprandial. Máximo } 4 \text { relajaciones transitorias del } \\
\text { LES (TLERs) con eructo durante los primeros } 10 \text { min posprandi- } \\
\text { ales, sin regurgitación de volumen, sin rumiación ni episodios } \\
\text { de eructo supragástrico. }\end{array}$ \\
\hline \multirow[t]{2}{*}{ Provocación farmacológica } & $\begin{array}{l}\text { Inhalador de nitrito de amilo (4-5 } \\
\text { inhalaciones) en posición reclinada }\end{array}$ & $\begin{array}{l}\text { Inhibición del músculo liso esofágico distal profundo y del } L E S \\
\text { con reducción en la IRP. En controles sanos, la IRP de la EGJ } \\
\text { inducida por nitrito de amilo es similar a la IRP deglutoria. } \\
\text { En pacientes con obstrucción por trastorno de la EGJ, como la } \\
\text { acalasia y la EGJOO funcional verdadera, la caída en la presión } \\
\text { de la EGJ inducida por el nitrito de amilo es mucho menor ( } \geq 10 \\
\mathrm{mmHg} \text { ) que la IRP deglutoria comprometida (es decir, ganancia } \\
\text { de relajación). En contraste, el nitrito de amilo en pacientes con } \\
\text { EGJOO secundaria a factores diferentes a disfunción del } \\
\text { músculo liso del } L E S \text {, muestra poco cambio en la presión de la } \\
\text { EGJ inducido por el nitrito de amilo }(<10 \mathrm{mmHg} \text { ). }\end{array}$ \\
\hline & $\begin{array}{l}\text { Colecistocinina (ССК) } 40 \mathrm{ng} / \mathrm{kg} / \mathrm{lV} \text { en } \\
\text { posición reclinada }\end{array}$ & $\begin{array}{l}\text { La CCK generalmente activa una respuesta motora bifásica en } \\
\text { el esófago. La fase } 1 \text { siempre está presente y empieza poco } \\
\text { después de la inyección. En controles sanos, la CCK induce un } \\
\text { acortamiento esofágico leve ( } 2 \mathrm{~cm} \text { o menos) asociado a } \\
\text { relajación incompleta de la EGJ (contracción inspiratoria del } \\
\text { diafragma crural preservada). } \\
\text { En contraste, en pacientes con disfunción inhibitoria como la } \\
\text { acalasia, la CCK induce una contracción paradójica de la EGJ } \\
\text { de más de } 50 \mathrm{mmHg} \text {. }\end{array}$ \\
\hline
\end{tabular}

La clasificación se basa en la posición primaria en la cual se administran 10 tragos húmedos, ya sea en supino o sedestación. La evaluación de degluciones en la posición secundaria y con provocación sirve como dato de apoyo (con excepción de la EGJ00 y la contractilidad ausente). DCI: integral de contractilidad distal; EGJ: unión esofagogástrica: EGJ00: obstrucción del flujo de salida de la EGJ; IRP: presión integrada de relajación; LES: esfínter esofágico inferior.

(>5 cm) en el frente de contracción. El límite superior normal de IRP para degluciones sólidas con el sistema Medtronic es de $25 \mathrm{mmHg}^{31}$. La asociación temporal entre las contracciones inefectivas y los síntomas del paciente (como disfagia) apoyan el diagnóstico de función peristáltica anormal clínicamente relevante ${ }^{11,30}$. 
- Provocación farmacológica: si está disponible en el laboratorio de motilidad, la provocación farmacológica con el protocolo de nitrito de amilo y/o colecistocinina puede ser útil para evaluar la fisiología en la EGJ. La Tabla 2 detalla el protocolo y las respuestas fisiológicas a la provocación farmacológica ${ }^{32,33}$.

\section{Consideraciones claves para el protocolo en el contexto de la Clasificación de Chicago}

Se espera que la mayoría de los Laboratorios sigan iniciando el protocolo de manometría en la posición de decúbito supino de forma convencional. Aunque el protocolo de HRM descrito se considera óptimo e incluyente, los profesionales pueden modificarlo para que se adapte a los recursos y el tiempo disponibles, siempre que se apliquen los valores normativos establecidos y que las otras posiciones y medidas de apoyo se utilicen adecuadamente. Los profesionales que decidan iniciar el estudio en la posición sedente deben realizar 10 degluciones en sedestación desde el principio.

La clasificación utilizada en CCv4.0 se basa en la administración de 10 tragos en la primera posición de la prueba, ya sea decúbito supino o sedestación. La valoración de las degluciones en la posición secundaria y con la provocación arroja datos de apoyo (excepto en casos de EGJOO y contractilidad ausente, como se detalla más adelante en este documento). La CCv4.0 reconoce la potencial variación de los hallazgos cuando se cambian las posiciones de prueba. Los hallazgos concordantes en la posición secundaria y con las pruebas provocadoras aumentan la confiabilidad de la clasificación y el diagnóstico final. Por el contrario, los hallazgos discordantes en la posición secundaria y/o con las pruebas provocadoras sugieren reconsiderar el diagnóstico y utilizar pruebas de apoyo antes de llegar a un diagnóstico conclusivo.

\section{CLASIFICACIÓN DE CHICAGO V4.0 PARA LAS ALTERACIONES DE LA MOTILIDAD ESOFÁGICA}

El esquema de clasificación jerárquica de las pasadas versiones de la Clasificación de Chicago se mantiene en la CCv4.0, por lo cual las alteraciones de la motilidad se clasifican como alteraciones del flujo de salida de la EGJ y/o alteraciones de la peristalsis (Tabla 3, Figura 3). Tal esquema de clasificación se aplica a los pacientes con anatomía normal del intestino delgado, sin intervenciones quirúrgicas o invasivas del mismo y sin hernia hiatal o hernia paraesofágica de gran tamaño. Dichos cambios anatómicos alteran la valoración de la métrica de la CC y se asocian con artificios de contacto por plegamiento del catéter que puede persistir después del cambio de posición. Además, en esos casos es crucial tener una endoscopia índice cuidadosa antes de la prueba manométrica, puesto que la evidencia endoscópica o radiográfica de obstrucción mecánica excluye el uso de CCv4.0. Adicionalmente, la CCv4.0 actualizada enfatiza que las alteraciones específicas de la motilidad se deben considerar clínicamente relevantes sólo en el contexto de síntomas compatibles y/o pruebas de apoyo, como se detalla en este documento y se indica con un asterisco en las figuras. 
TABLA 3. Clasificación y definición de las alteraciones manométricas

\begin{tabular}{|c|c|c|}
\hline Clasificación & Trastorno & Definición \\
\hline \multirow{4}{*}{$\begin{array}{l}\text { Alteraciones del flujo } \\
\text { de salida de la } E G J\end{array}$} & Acalasia tipo I & IRP mediana anormal y peristalsis del todo fallida \\
\hline & Acalasia tipo II & $\begin{array}{l}\text { IRP mediana anormal, peristalsis completamente fallida, y presurización panesofági- } \\
\text { ca en } \geq 20 \% \text { de las degluciones }\end{array}$ \\
\hline & Acalasia tipo III ${ }^{\mathrm{a}}$ & $\begin{array}{l}\text { IRP mediana anormal y contracción prematura/espástica en } \geq 20 \% \text { de las deglu- } \\
\text { ciones sin evidencia de peristalsis }\end{array}$ \\
\hline & $\begin{array}{l}\text { Obstrucción del flujo de salida de } \\
\text { la } E G J^{j, c}\end{array}$ & $\begin{array}{l}\text { IRP mediana anormal (en supino y sedestación), presión intrabolo elevada } \geq 20 \% \\
\text { (supino), y no se cumplen criterios para acalasia }\end{array}$ \\
\hline \multirow{4}{*}{$\begin{array}{l}\text { Alteraciones de la } \\
\text { peristalsis }\end{array}$} & Contractilidad ausente & IRP mediana normal (en supino y sedestación) y peristalsis del todo fallida \\
\hline & Espasmo esofágico distalc & IRP mediana normal y contracción prematura/espástica en $\geq 20 \%$ de las degluciones \\
\hline & Esófago hipercontráctil ${ }^{c}$ & IRP mediana normal y degluciones hipercontráctiles $\geq 20 \%$ \\
\hline & Motilidad esofágica inefectiva & IRP mediana normal, con degluciones inefectivas $\geq 70 \%$ o peristalsis fallidas $\geq 50 \%$ \\
\hline \multicolumn{3}{|c|}{ 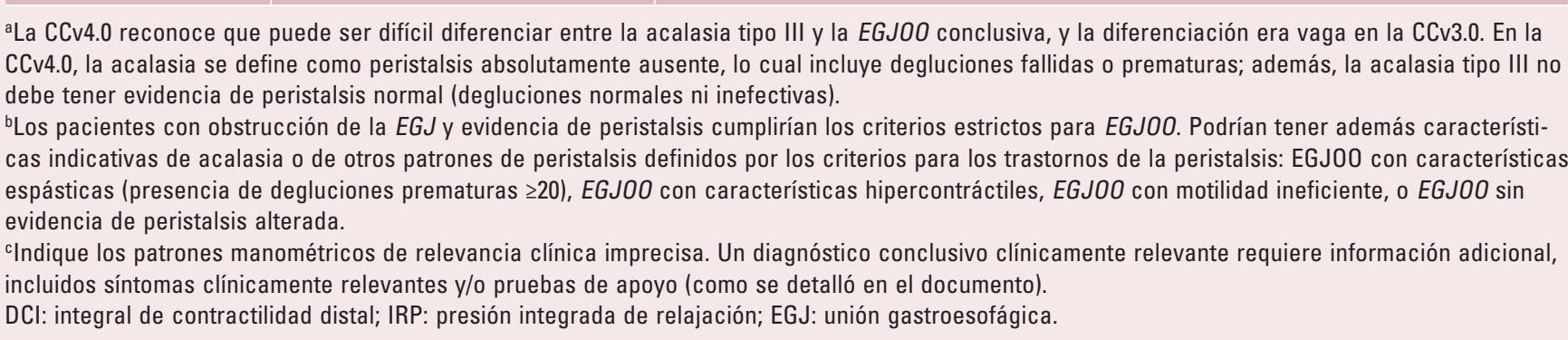 } \\
\hline
\end{tabular}

\section{ALTERACIONES DEL FLUJO DE SALIDA DE LA EGJ (TABLA S2)}

En concordancia con versiones previas de la Clasificación de Chicago, las alteraciones del flujo de salida de la EGJ incluyen acalasia (tipos I, II, y III) y obstrucción del flujo de salida de la EGJ. Para el diagnóstico de cualquiera de las alteraciones del flujo de salida de la EGJ se requiere una IRP mediana anormal en la posición primaria. Dada la alta probabilidad preprueba de acalasia con la exigencia de peristalsis 100\% ausente, una IRP anormal en la posición primaria se considera conclusiva de acalasia. De otro lado, la presencia de peristalsis apreciable en el EGJOO disminuye la probabilidad preprueba de verdadera disfunción del LES. Entonces, para el diagnóstico manométrico conclusivo de EGJOO se requiere una IRP mediana anormal tanto en la posición primaria como en la secundaria, así como pruebas complementarias que confirmen el diagnóstico.

\section{ACALASIA}

La CCv4.0 mantiene tres subtipos de acalasia: el tipo I, semejante a la acalasia clásica, el tipo II que se observa con presurización panesofágica, y el tipo III, o acalasia espástica (Figura 4). Como se mencionó anteriormente, una actualización de la $\mathrm{C} C \mathrm{~V} 4.0$ respecto a acalasia es que la IRP mediana anormal se debe observar en la posición primaria, ya sea decúbito supino o sedestación (si se realiza con 10 degluciones). No se requiere que la IRP mediana sea anormal en ambas 


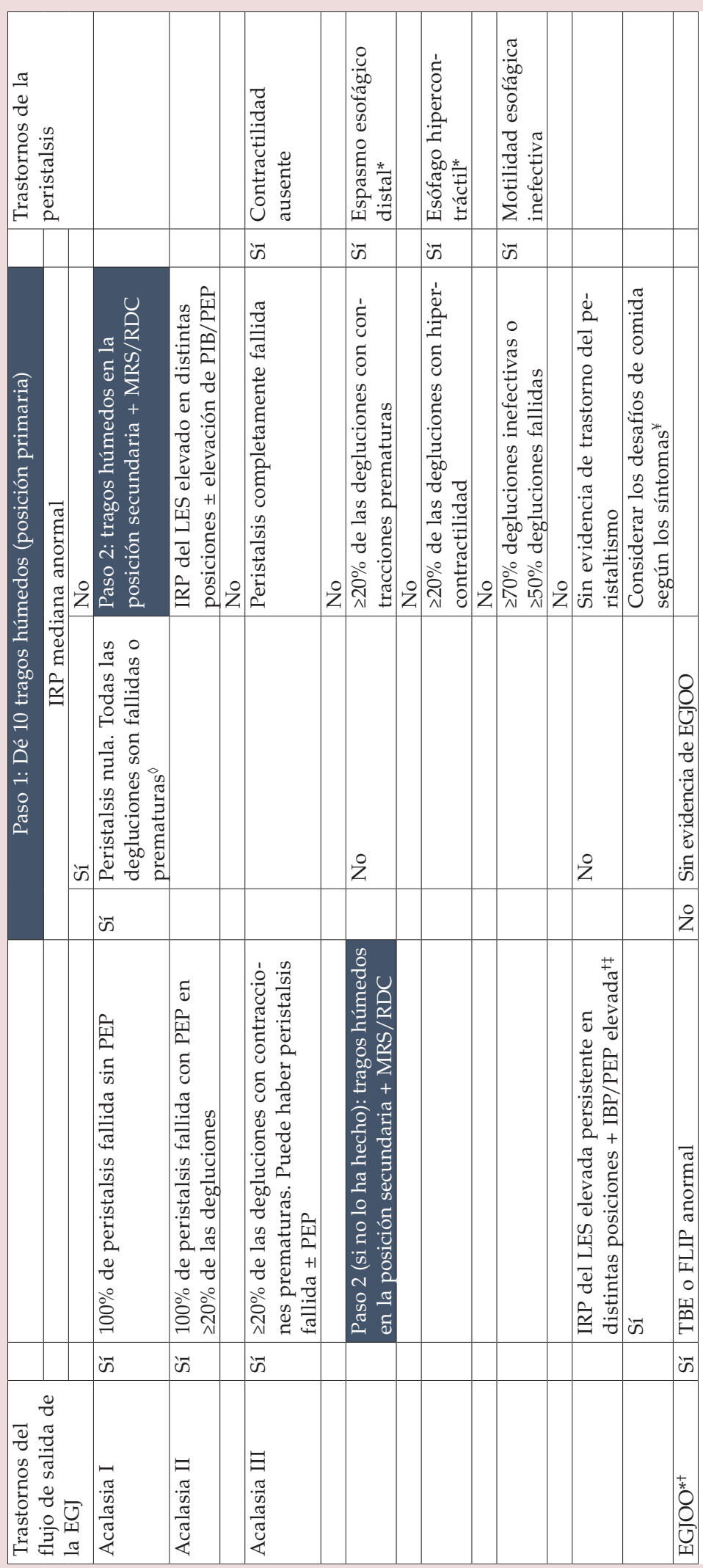

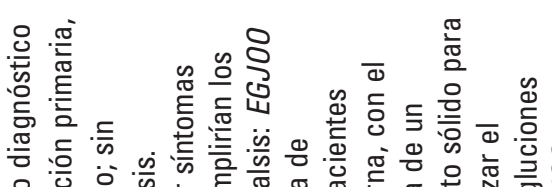

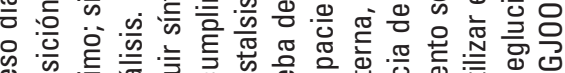

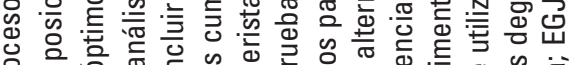

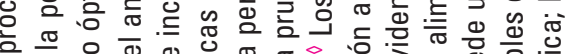

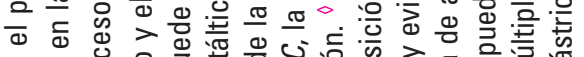
\&

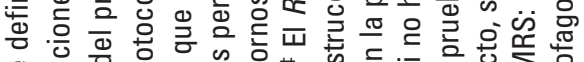

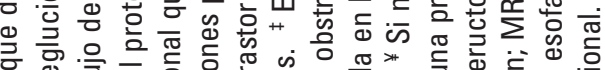

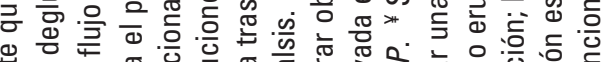

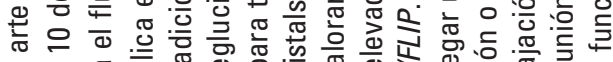

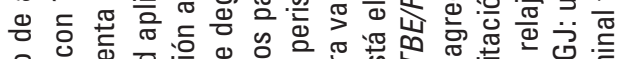
음 0 \% :

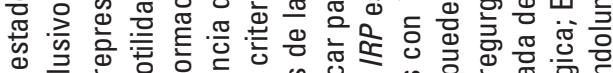
ब ठ 己

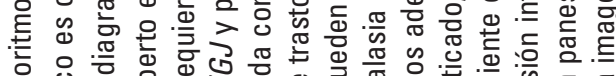

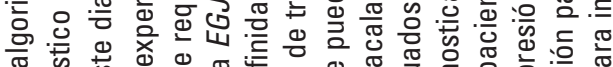

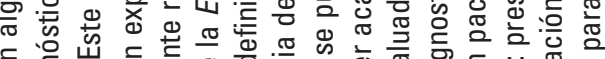

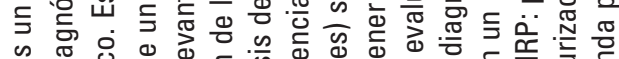

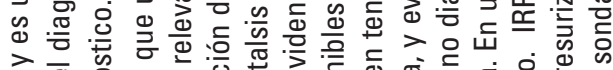

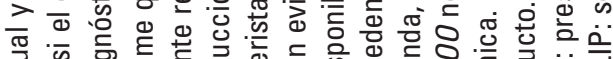

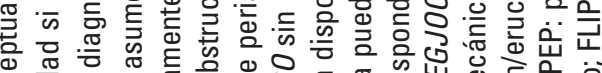
类

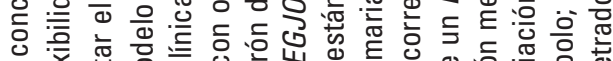
语高 造类

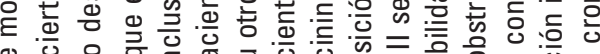

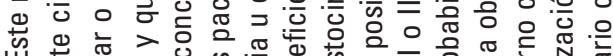

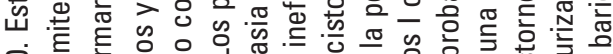

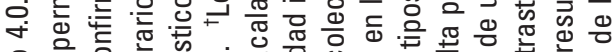
过 $\frac{0}{0}$ 능

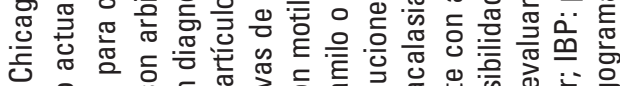

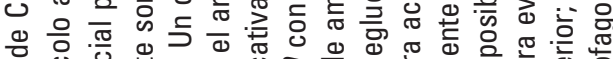

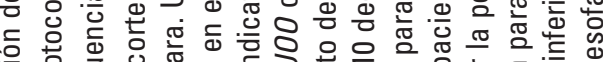
次 은

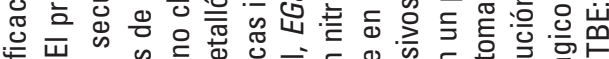

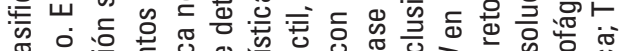
元 응

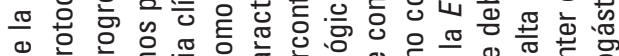

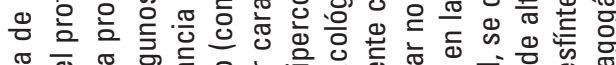

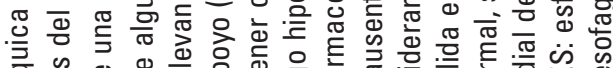
혼

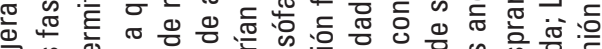

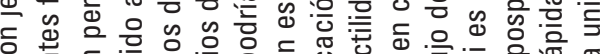

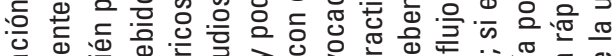
元造

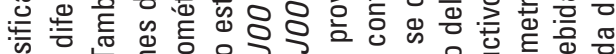
元

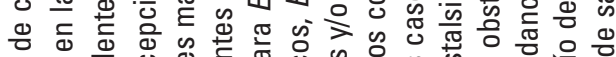

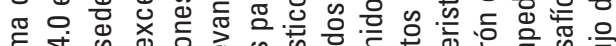

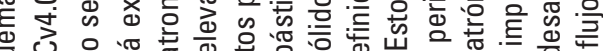

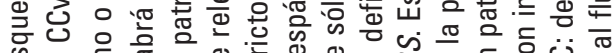

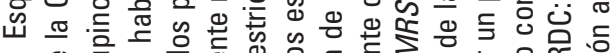
m 死

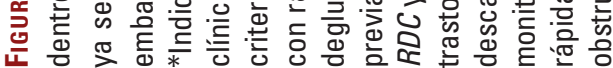


posiciones. Más aún, en la CCv4.0 la definición de acalasia exige peristalsis absolutamente ausente, definida como la presencia de peristalsis fallida o contracción prematura en todas las degluciones.

\section{Diagnóstico conclusivo de acalasia}

Acalasia tipo I: el diagnóstico conclusivo de acalasia tipo I se define como una IRP mediana anormal y contractilidad ausente (peristalsis completamente fallida) (GRADE muy bajo, recomendación fuerte) ${ }^{22,34-37}$.

Acalasia tipo II: el diagnóstico conclusivo de acalasia tipo II se define como una IRP mediana anormal y contractilidad ausente (peristalsis fallida por completo) con presurización panesofágica en $20 \%$ de las degluciones o más (GRADE muy bajo, recomendación fuerte) $)^{22,34-37}$.

Acalasia tipo III: el diagnóstico conclusivo de acalasia tipo III se define como IRP mediana anormal y evidencia de espasmo (20\% de las degluciones o más con contracción prematura) sin evidencia de peristalsis (GRADE muy bajo, recomendación fuerte $)^{22,34-37}$.

\section{Diagnóstico no conclusivo de acalasia}

- Un diagnóstico no conclusivo de acalasia tipos I o II incluye contractilidad ausente sin peristalsis apreciable, con valores de IRP normales o en el límite superior en ambas posiciones, con o sin presurización panesofágica en $20 \%$ de las degluciones o más (recomendación fuerte) (Figura 5).
- La evidencia de peristalsis apreciable con el cambio de posición en presencia de un patrón de acalasia tipos I o II en la posición primaria puede cambiar el diagnóstico hacia no conclusivo, requiriendo estudios de apoyo (observación clínica aceptada).

- Un diagnóstico no conclusivo de acalasia tipo III incluye una IRP anormal con evidencia de espasmo y peristalsis. Si estos casos cumplen estrictamente los criterios de EGJOO (como se especificó en la sección de $E G J)$, estos pacientes se deben clasificar como EGJOO con características espásticas, lo cual puede representar una variante de acalasia.

\section{Consideraciones adicionales para acalasia}

- El punto de corte para los espasmos en $20 \%$ es arbitrario. Se puede aumentar la confianza en el diagnóstico de la acalasia tipo III con un mayor número de degluciones prematuras o espásticas.

- En los pacientes con diagnóstico no conclusivo de acalasia en presencia de disfagia como síntoma de presentación, se deben realizar pruebas de apoyo con un TBE, preferiblemente en conjunto con un trago de tableta de bario, y/o prueba con FLIP (GRA$D E$ muy bajo, recomendación fuerte) $)^{13,14,38-44}$.

- El uso de opioides se asocia con acalasia tipo III, por lo tanto, el estudio se debe realizar sin que el paciente consuma medicamentos opioides, si es posible (GRADE bajo, recomendación condicional) ${ }^{45,46}$. El momento para descontinuar el opioide se define según sea la vida media del medicamento.

Los siguientes conceptos sobre acalasia no satisficieron los criterios de nivel de acuerdo, pero 

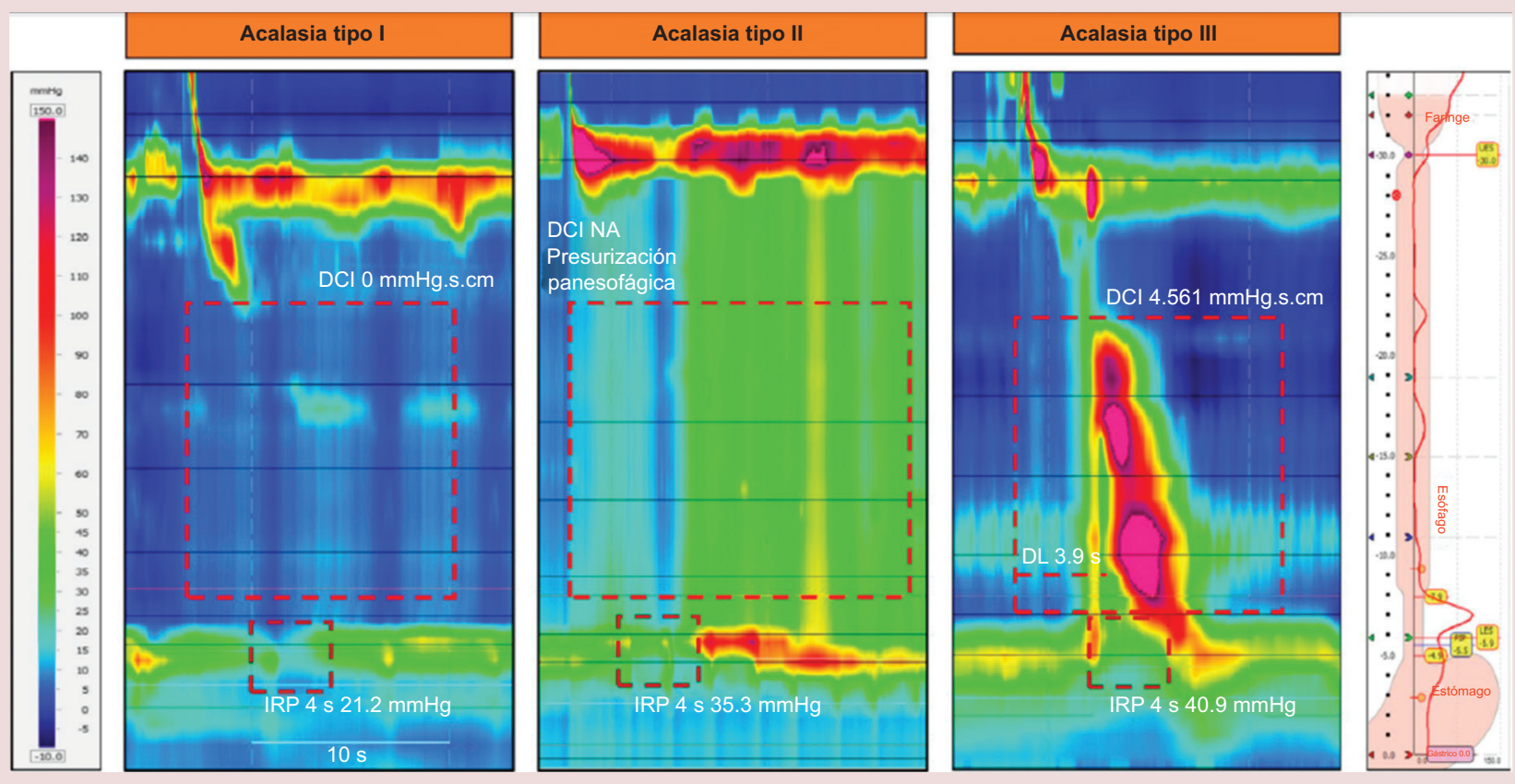

Figura 4. Subtipos de acalasia.

Acalasia tipo I: la presión integrada de relajación (IRP) está elevada con peristalsis fallida (integral de contractilidad distal, $D C l<100$ mmHg-s-cm), y sin presurización panesofágica.

Acalasia tipo II: la IRP está elevada con peristalsis fallida y presurización panesofágica.

Acalasia tipo III: la IRP está elevada con una $D C$ I normal, y una latencia distal reducida.

NA: no aplica. Cortesía de University of California San Diego Center for Esophageal Diseases.

son consideraciones recomendadas para las HRM sugestivas de acalasia, como se detalla más adelante en la revisión técnica. El valor de corte de la presurización esofágica pretende ser una guía más que un umbral rígido. La distinción entre acalasia tipos I y II es algo arbitraria y no tiene implicaciones clínicas importantes más allá de los niveles muy bajos (típicamente $<15 \mathrm{mmHg}$ ) presentes en la dilatación esofágica moderada a grave y una incapacidad para generar presurización de vaciado. Los pacientes con valores de presurización panesofágica $>70 \mathrm{mmHg}$ pueden tener un espasmo incorporado, lo cual puede tener un efecto en el resultado clínico.

\section{OBSTRUCCIÓN DEL FLUJO DE SALIDA DE LA UNIÓN GASTROESOFÁGICA}

Una actualización crítica en CCv4.0 es la clarificación y definición rigurosa de la EGJOO (Figura 6). Luego de que la EGJOO se incluyera como una alteración de la motilidad, se identificó un patrón de motilidad correspondiente a EGJOO en cerca del 10\% de los pacientes llevados a $H R M^{19,47}$. Mientras que una proporción de EGJOO puede evolucionar hacia la acalasia o representar una variante de acalasia, más de un tercio de los casos puede ser clínicamente irrelevante y relacionado con etiologías benignas, incluyendo efectos mecánicos, uso de opioides, y artificios, como se 

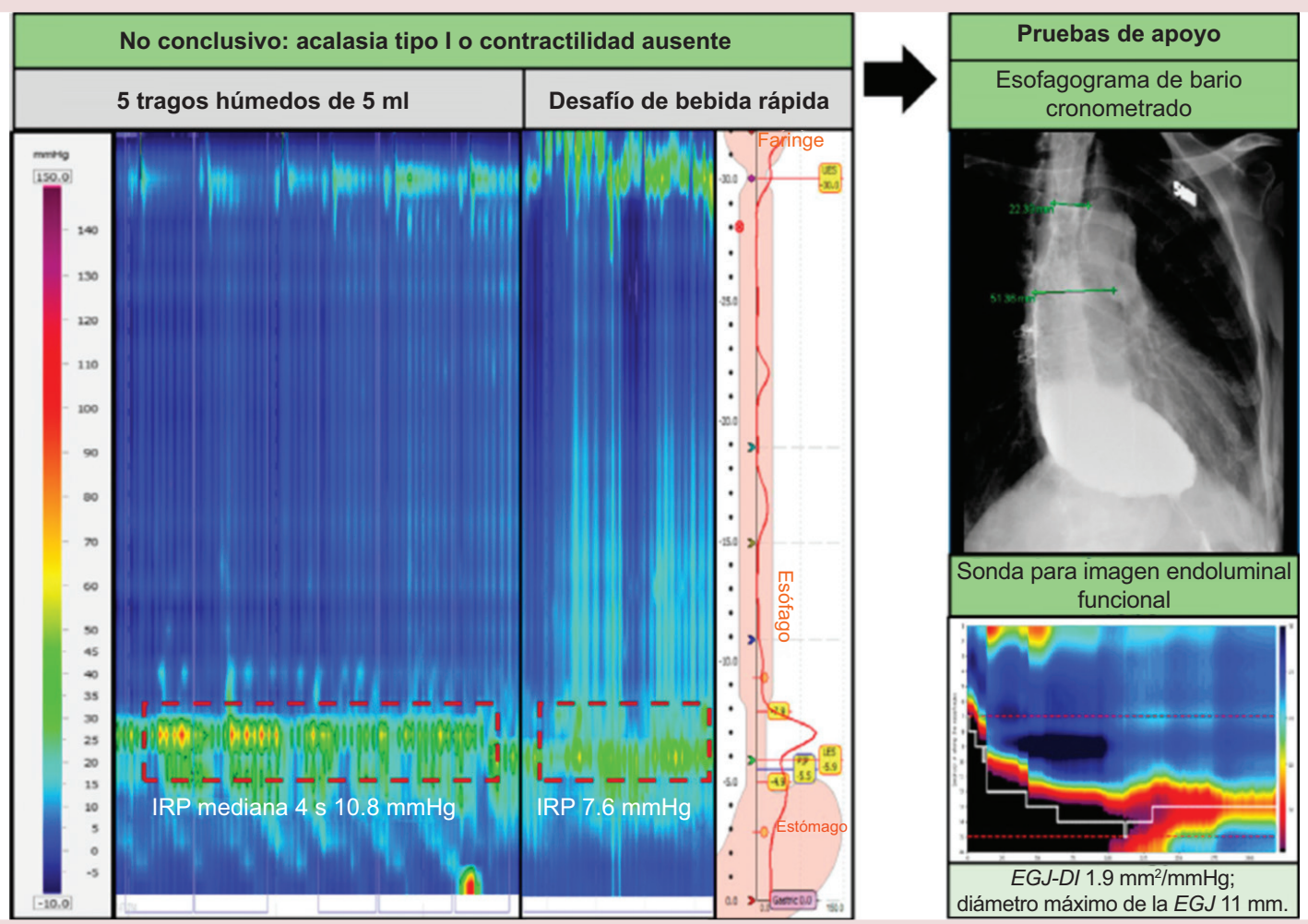

Figura 5. El diagnóstico no conclusivo de acalasia o contractilidad ausente requiere pruebas de apoyo. Los hallazgos son no conclusivos para acalasia tipo I o contractilidad ausente puesto que hay peristalsis completamente fallida pero la presión integrada de relajación (IRP) está en el límite superior o normal con tomas húmedas de $5 \mathrm{ml}$. Con el reto de bebida rápida, no hay inhibición deglutoria a lo largo del esfínter esofágico inferior (LES). En el escenario de hallazgos no conclusivos se requieren pruebas de apoyo, como esofagograma de bario cronometrado y/o prueba funcional con la sonda para imagen endoluminal funcional (FLIP). En la imagen, el esofagograma de bario cronometrado demostró un esófago distal dilatado con retención de bario. En la FLIP, el índice de distensibilidad de la EGJ (EGJ-DI) está reducido, el diámetro máximo del EGJ está reducido y no hay respuesta contráctil a la distensión. Cortesía de Northwestern Esophageal Center.

describe en la subsiguiente revisión técnica de EGJOO. Para evitar tratamientos innecesarios y optimizar los resultados, es crítico aclarar cuáles pacientes con EGJOO manométrica tienen una fisiología obstructiva causante de síntomas y requieren intervención ${ }^{14,19,23,24,40,47-53}$. Por lo tanto, la CCv4.0 plantea las siguientes recomendaciones para distinguir entre la EGJOO clínicamente relevante que puede representar una alteración motora patológica respondedora al tratamiento, y una observación manométrica clínicamente irrelevante.

\section{Diagnóstico conclusivo clínicamente relevante de EGJOO}

- El diagnóstico manométrico de EGJOO siempre se considera clínicamente no conclusivo (recomendación fuerte).

- El diagnóstico manométrico de EGJOO se define como la IRP mediana elevada en las posiciones primaria y secundaria y la presión intrabolo elevada en $\geq 20 \%$ de las degluciones en la posición supina, con evidencia de peristalsis (GRADE 


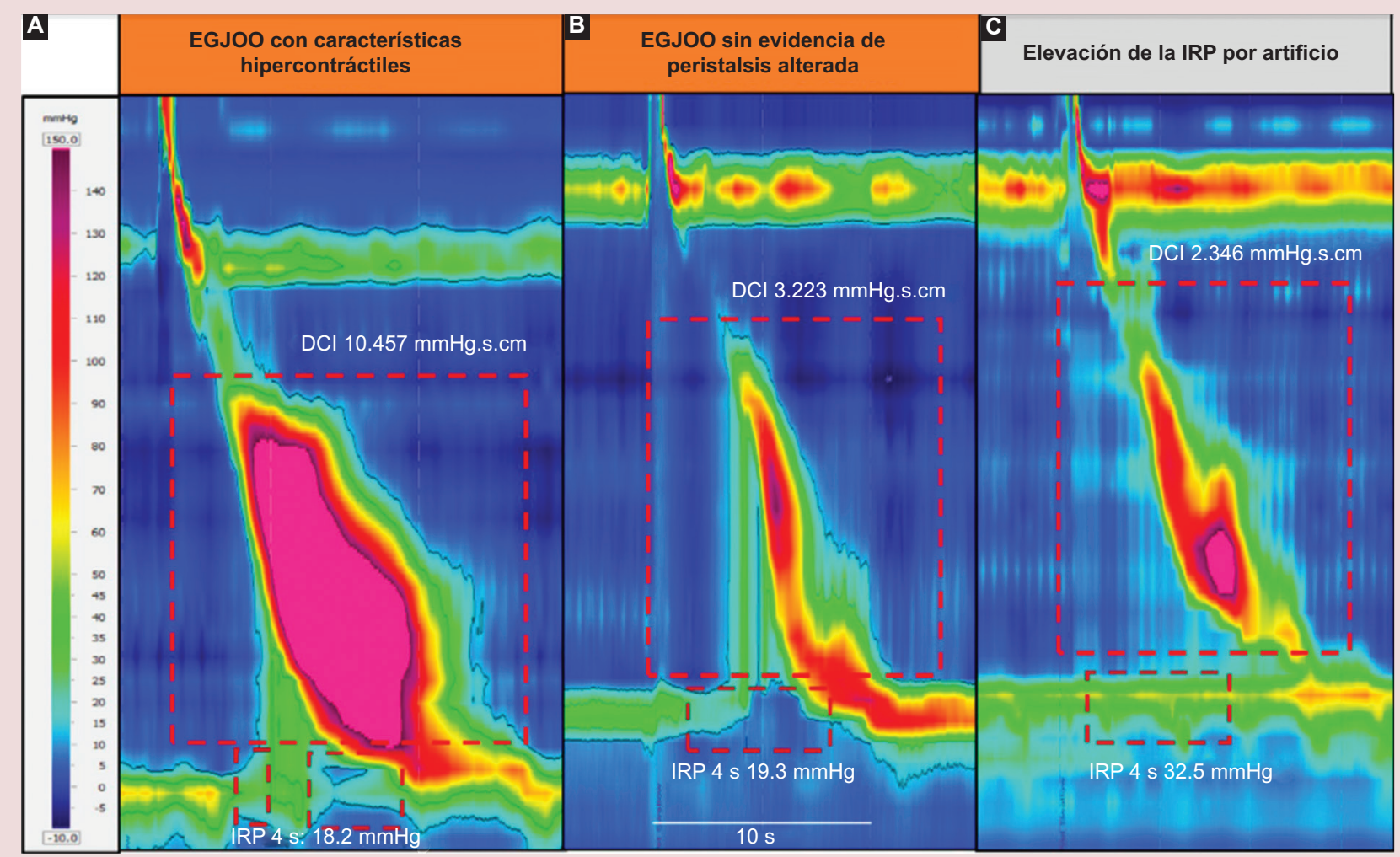

Figura 6. Subtipos de EGJOO. A: EGJOO con características hipercontráctiles; IRP elevada con presurización intrabolo y deglución hipercontráctil. B: EGJOO sin evidencia de peristalsis alterada; la IRP elevada con vigor contráctil normal. C: EGJOO manométrica relacionada con elevación de la IRP por artificio; la IRP está elevada en ausencia de presurización intrabolo, probablemente se asocia a un artificio. Cortesía de National Jewish Health, Esophageal Function Laboratory. EGJ: unión esofagogástrica; EGJ00: obstrucción del flujo de salida de la EGJ; IRP: presión integrada de relajación; DCI: integral de contractilidad distal.

bajo, recomendación condicional) $12,19,47,51,52,54-56$.

- El diagnóstico conclusivo clínicamente relevante de EGJOO requiere un diagnóstico manométrico de EGJOO y síntomas clínicamente relevantes, con al menos una de las siguientes pruebas que apoyen obstrucción: TBE, preferiblemente junto con un trago de tableta de bario y/o prueba con FLIP (GRADE moderado, recomendación condicional) ${ }^{12-14,19,38}$.

- Los síntomas clínicamente relevantes de EGJOO incluyen disfagia y dolor torácico no cardiogénico (GRADE bajo, recomendación condicional) 24,40,48,50-52,57,58.

\section{Diagnóstico no conclusivo de EGJOO}

- Los hallazgos anormalmente elevados aislados no son conclusivos para el diagnóstico manométrico de EGJOO. Éstos incluyen elevación aislada de la IRP en supino, elevación aislada de la IRP en sedestación, o elevación aislada de la presión intrabolo en supino (GRADE bajo, recomendación fuerte $)^{59,60}$. 


\section{Consideraciones adicionales para EGJ00}

Las siguientes condiciones no son esenciales pero aportan evidencia de apoyo para el diagnóstico de EGJOO.

- La evidencia que apoya el diagnóstico manométrico de EGJOO incluye obstrucción al flujo de salida y presurización esofágica durante el RDC (GRADE muy bajo, recomendación condicional) ${ }^{10,11,28-31,47 .}$

- La evidencia que apoya el diagnóstico manométrico de EGJOO incluye obstrucción al flujo de salida durante la prueba de alimento sólido, especialmente si hay asociación temporal con los síntomas del paciente (recomendación condicional) ${ }^{11,31}$ (Figura S1).

- La evidencia que apoya el diagnóstico manométrico de EGJOO incluye función anormal de la EGJ luego de provocación farmacológica ${ }^{12}$ (recomendación condicional) (Figura S2).

La EGJOO se debe describir dentro del contexto del patrón de peristalsis: EGJOO con características espásticas (características de acalasia tipo III), EGJOO con características de hipercontractilidad, EGJOO con motilidad inefectiva, o EGJOO sin evidencia de peristalsis alterada (observación clínica aceptada).

\section{ALTERACIONES DE LA PERISTALSIS (TABLA S3)}

En concordancia con versiones previas de la Clasificación de Chicago, la contractilidad ausente, el espasmo esofágico distal (DES), el esófago hipercontráctil y la IEM se consideran alteraciones de la peristalsis. La peristalsis fragmentada ahora se excluyó de la lista de alteraciones y se incorporó en el diagnóstico general de IEM (GRADE muy bajo, recomendación fuerte $)^{61,62}$. Las alteraciones de la peristalsis se consideran cuando se ha descartado una alteración del flujo de salida de la EGJ. Puede existir una superposición de las características de peristalsis anormal. En estos escenarios, se debe emplear una aproximación jerárquica a la clasificación diagnóstica con DES primero, esófago hipercontráctil después, y finalmente IEM, con un comentario que manifieste la presencia de las características que se superponen.

Cabe resaltar nuevamente que la determinación diagnóstica se debe basar en la posición primaria en la cual se dan 10 tragos, ya sea en supino o sedestación. La valoración de las degluciones en la posición secundaria y con la provocación proporciona evidencia de apoyo. La concordancia de la clasificación peristáltica con los cambios de posición fortalece la confianza en la clasificación y el diagnóstico clínico final. La discordancia, por el contrario, debe promover la reconsideración de más pruebas de apoyo.

Una actualización importante en la CCv4.0 es el reconocimiento de DES y el esófago hipercontráctil como patrones manométricos que no siempre equivalen a enfermedad clínica, en semejanza con los conceptos que subyacen a la EGJOO. Según la CCv4.0, estas alteraciones de la peristalsis son clínicamente relevantes sólo en el contexto clínico apropiado y cuando están apoyadas por estudios adicionales, como se detalló en esta sección. 


\section{CONTRACTILIDAD AUSENTE}

Los criterios diagnósticos de contractilidad ausente no se revisaron en la CCv4.0.

Un diagnóstico conclusivo de contractilidad ausente se define como la IRP mediana normal en las posiciones supina y sedente, $\mathrm{y}$ peristalsis absolutamente fallida $(D C I<100$ $\mathrm{mmHg} \bullet \mathrm{s} \bullet \mathrm{cm}$ ) (observación clínica aceptada) (Figura 7).

En el contexto de contractilidad ausente, los valores de IRP mediana limítrofes, particularmente la IRP mediana en posición supina de 10 a 15 mmHg con el sistema Meditronic, deben generar la consideración de acalasia tipo I. Como se discutió en la sección de acalasia, si la disfagia es el síntoma predominante, se deben considerar estudios de apoyo con $T B E$, preferiblemente incluyendo un trago de tableta de bario y una prueba con FLIP (Figura 5).

\section{ESPASMO ESOFÁGICO DISTAL (DES)}

El DES describe un patrón motor esofágico anormal caracterizado por contracciones espásticas o prematuras en el esófago distal (Figura 8). El DES se define como contracciones con una latencia distal menor a 4.5 segundos, en el escenario de un DCI mayor a $450 \mathrm{mmHg} \bullet \mathrm{s}^{\bullet} \mathrm{cm}$. El DES manométrico puede tener significancia clínica variable y, en consecuencia, una actualización del CCv4.0 es la distinción entre DES clínicamente relevante y observaciones manométricas clínicamente irrelevantes.

\section{Diagnóstico clínicamente relevante de espasmo esofágico distal}

- Un diagnóstico clínicamente relevante de $D E S$ requiere tanto síntomas clínicamente relevantes como un diagnóstico manométrico conclusivo de DES (GRADE bajo, recomendación condicional $)^{20}$.

- Un diagnóstico manométrico conclusivo de DES se define como la presencia de al menos $20 \%$ de contracciones prematuras (GRADE bajo, recomendación fuerte $)^{20}$.

- Los síntomas de DES clínicamente relevantes incluyen disfagia y dolor torácico no cardiogénico (observación clínica aceptada).

\section{Diagnóstico no conclusivo de espasmo esofágico distal}

- La presencia de latencia distal reducida ( $D L<4.5$ segundos) en al menos el $20 \%$ de las contracciones pero con un DCI $<450 \mathrm{mmHg} \bullet \mathrm{s}^{\bullet} \mathrm{cm}$ no es conclusiva para un diagnóstico manométrico de DES (GRA$D E$ bajo, recomendación condicional $)^{20}$.

El grupo de trabajo CCv4.0 reconoce que el $C D P$ puede ser difícil de identificar. En este escenario, hay que considerar métodos alternativos para diagnosticar DES (recomendación fuerte). Esto se trata con mayor detalle en la sección previa sobre métricas y será discutido más adelante en la subsiguiente revisión técnica de DES.

\section{ESÓFAGO HIPERCONTRÁCTIL}

El esófago hipercontráctil describe una anomalía manométrica específica definida por 

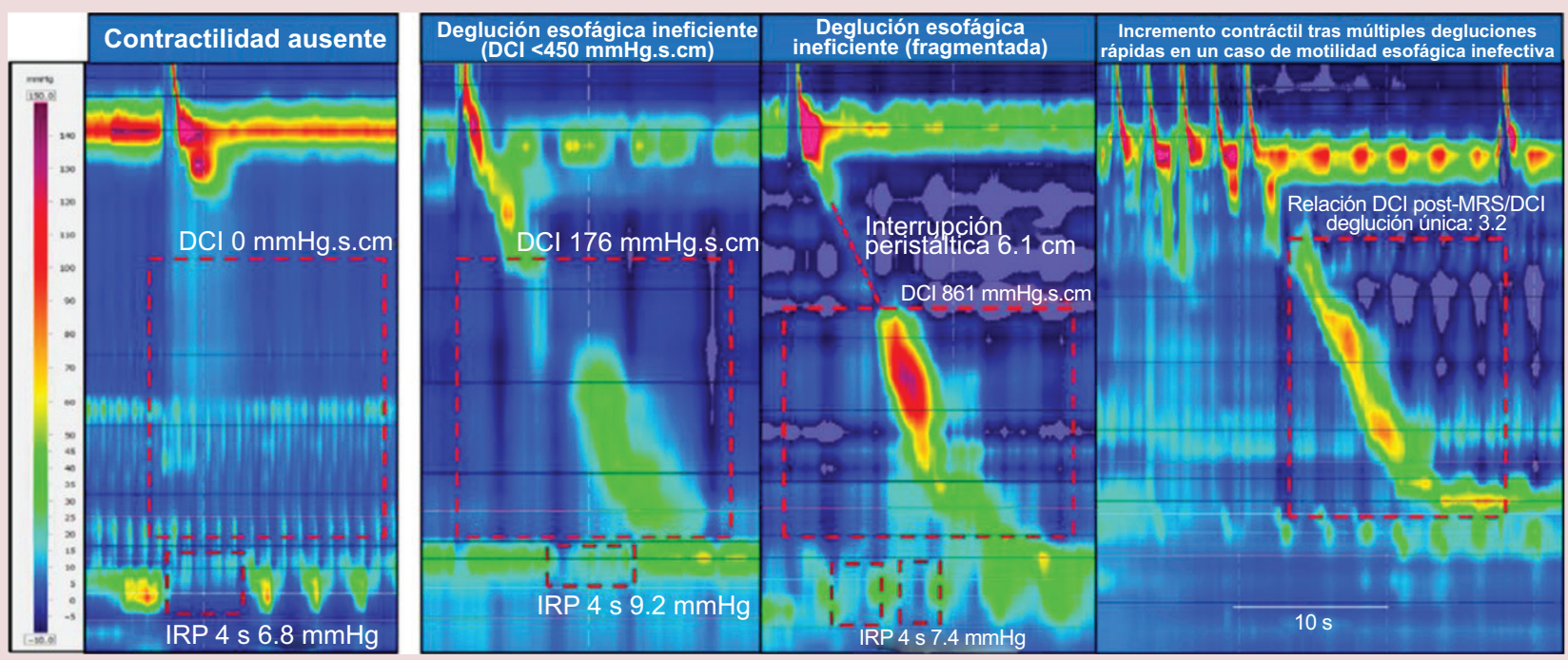

Figura 7. Trastornos de la peristalsis con vigor contráctil reducido o integridad de la peristalsis. Éstos incluyen contractilidad ausente o motilidad esofágica ineficiente (relacionada con vigor contráctil reducido o con peristalsis fragmentada). En este ejemplo de contractilidad ausente hay una peristalsis fallida con una IRP normal. En el primer ejemplo de IEM, la $D C I$ está reducida con $I R P$ normal. En el segundo ejemplo de $I E M$, la $D C l$ es normal con una fragmentación de la peristalsis $>5 \mathrm{~cm}$ en el escenario de una $I R P$ normal. Durante las múltiples degluciones rápidas $(M R S)$ no hay actividad contráctil y sí inhibición deglutoria del esfínter esofágico inferior seguida de una $D C /$ mayor que la $D C l$ de deglución única, lo que significa un incremento contráctil intacto. Cortesía de University of California San Diego Center for Esophageal Diseases.

vigor peristáltico excesivo, lo cual puede incluir poscontracción excesiva del LES no asociada con obstrucción mecánica (Figura 8) (GRADE muy bajo, recomendación

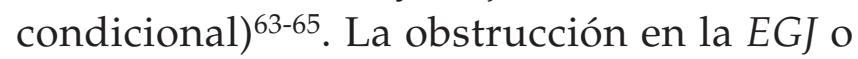
en el esófago distal puede inducir una respuesta hipercontráctil, por tanto es crucial descartar tal obstrucción antes de considerar el diagnóstico del esófago hipercontráctil. Los patrones motores heterogéneos pueden cumplir criterios manométricos para esófago hipercontráctil y tienen significado clínico variable. Entonces, tal y como con el DES, una actualización importante en CCv4.0 es la distinción entre el esófago hipercontráctil clínicamente significativo y las observaciones manométricas clínicamente irrelevantes.

\section{Diagnóstico conclusivo clínicamente relevante de esófago hipercontráctil}

- Un diagnóstico clínicamente relevante de esófago hipercontráctil requiere tanto síntomas clínicamente relevantes como un diagnóstico manométrico conclusivo de esófago hipercontráctil (GRADE muy bajo, recomendación fuerte $)^{57,63,65,66}$.

- Un diagnóstico manométrico conclusivo de esófago hipercontráctil se define como degluciones hipercontráctiles en supino de un $20 \%$ o más (GRADE muy bajo, recomendación condicional) ${ }^{63-65}$.

- Los síntomas clínicamente relevantes de esófago hipercontráctil incluyen disfagia y dolor torácico no cardiaco (GRADE muy bajo, recomendación fuerte $)^{57,63,65,66}$. 

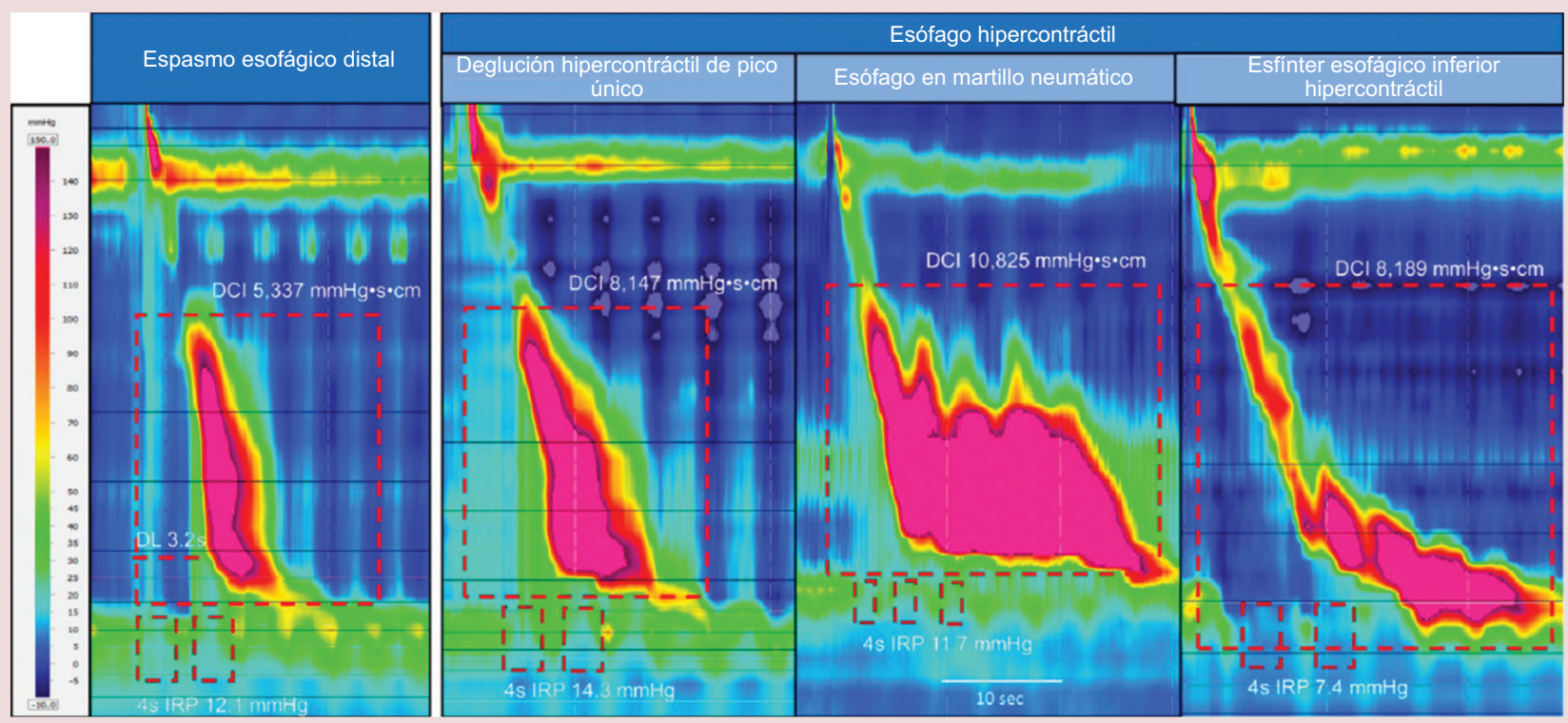

Figura 8. Trastornos de la peristalsis con espasticidad esofágica o hipercontractilidad. Éstos incluyen espasmo esofágico distal o esófago hipercontráctil. En este ejemplo de espasmo esofágico distal, la $D C l$ es normal con una latencia distal reducida y una $I R P$ normal. El esófago hipercontráctil incluye subgrupos: deglución hipercontráctil de pico único, esófago hipercontráctil en martillo neumático, y esófago hipercontráctil con poscontracción del LES. Cortesía de University of Carlifornia San Diego Center for Esophageal Diseases; Northwestern Esophageal Center, y Mayo Clinic Arizona Motility Lab.

IRP: presión integrada de relajación; DCI: integral de contractilidad distal; LES: esfínter esofágico inferior.

\section{Consideraciones adicionales para el esófago hipercontráctil}

El diagnóstico de esófago hipercontráctil puede hacerse sólo cuando no se cumplen los criterios para acalasia o espasmo esofágico distal y se ha descartado cuidadosamente una obstrucción mecánica (GRADE muy bajo, recomendación fuerte) ${ }^{63-65}$.

Dada la heterogeneidad de los patrones hipercontráctiles, el grupo promovió una aproximación cautelosa en términos de tratar el vigor contráctil como un punto extremo. El grupo aconsejó un tratamiento médico conservador antes de considerar una intervención quirúrgica o endoscópica. El subgrupo de trabajo de esófago hipercontráctil también propuso conceptos que no satisficieron los criterios para nivel de acuerdo, los cuales están detallados en la revisión técnica subsiguiente. Hay tres subgrupos generales de esófago hipercontráctil: deglución hipercontráctil de pico único, esófago en martillo neumático con contracciones repetitivas prolongadas (especialmente en la fase pospico), y degluciones hipercontráctiles con una poscontracción vigorosa del LES. El subgrupo de esófago en martillo típicamente se asocia con valores DCI más altos y mayor gravedad de los síntomas ${ }^{67-69}$. El grupo de trabajo de CCv4.0 en conjunto reconoce la necesidad crítica de mayor investigación para una mejor caracterización de los fenotipos fisiológicos y los resultados clínicos en el esófago hipercontráctil. 


\section{MOTILIDAD ESOFÁGICA INEFECTIVA}

Las versiones previas de la Clasificación de Chicago categorizaron la IEM y la peristalsis fragmentada como alteraciones menores de la motilidad. En la CCv4.0 la peristalsis fragmentada se incluye bajo la definición de IEM. Además, los criterios diagnósticos para IEM se han hecho más rigurosos, en correspondencia con los datos emergentes. Como resultado de estos cambios en la definición de IEM y peristalsis fragmentada, la CCv4.0 no hace distinción entre alteraciones menores y mayores (Figura 7).

\section{Diagnóstico conclusivo de motilidad esofágica inefectiva}

- Un diagnóstico conclusivo de IEM requiere más del 70\% de degluciones inefectivas o al menos $50 \%$ de peristalsis fallidas (GRA-

$D E$ muy bajo, recomendación fuerte) $)^{61,70-72}$. Como se detalló en Umbrales Diagnósticos y en la Tabla 1, una deglución inefectiva incluye una contracción débil $\left(D C I \geq 100 \mathrm{mmHg} \bullet \mathrm{s}^{\bullet} \mathrm{cm}\right.$ $\left.\mathrm{y}<450 \mathrm{mmHg} \bullet \mathrm{s}^{\bullet} \mathrm{cm}\right)$, peristalsis fallida (DCI $\left.<100 \mathrm{mmHg} \bullet \mathrm{s}^{\bullet} \mathrm{cm}\right)$, o deglución fragmentada.

\section{Diagnóstico no conclusivo de motilidad esofágica inefectiva}

- La presencia de 50 a 70\% de degluciones inefectivas no es conclusiva para diagnóstico de IEM. En estos casos, las pruebas de apoyo fortalecerán la confianza en el diagnóstico de IEM (GRADE muy bajo, recomendación condicional $)^{62,73}$.

\section{Consideraciones adicionales sobre la motilidad esofágica inefectiva}

Las siguientes condiciones no se requieren para definir IEM, pero pueden proporcionar evidencia de apoyo:

- La evidencia de apoyo para el diagnóstico de IEM incluye pobre tránsito del bolo en el esofagograma de impedancia o con bario (GRA$D E$ muy bajo, recomendación condicional) $)^{74-76}$.

- La evidencia de apoyo para el diagnóstico de IEM incluye falta de reserva de contracción en la prueba de MRS (GRADE muy bajo, recomendación condicional) ${ }^{26,77}$.

\section{MÉTRICA DE LA UNIÓN GASTROESOFÁGICA}

Una ventaja de la topografía de presión esofágica de alta resolución disponible hoy en día sobre el trazado lineal convencional es la capacidad de calcular con precisión la función de barrera de la EGJ en reposo incluyendo la relación entre el LES, el diafragma crural $(C D)$, y el punto de inversión respiratoria $(R I P)$. Otro parámetro incluido en la prueba es la integral de contractilidad de la EGJ (EGJ$C I)$, una medida de la contractilidad de la $E G J$ en relación con la respiración. De esta manera, una prioridad de la CCv4.0 fue proveer una guía para posibilitar una mejor caracterización del complejo $E G J$ durante un registro basal en la posición primaria.

- En el registro basal se debe medir el complejo EGJ con la respiración calmada en un segmento relativamente desprovisto de deglución y/o artificios de registro. Esta recomendación también hace referencia a la medición de la presión intragástrica, que debería ser medida 
por debajo del $C D$ por tres ciclos respiratorios completos, preferiblemente en el mismo segmento que se usó para medir la EGJ-CI (recomendación fuerte).

- El RIP es la localización axial en la cual el cambio inspiratorio en la presión hace transición desde un incremento inspiratorio característico de los registros intraabdominales, hacia un descenso inspiratorio característico de los registros intratoráci$\cos$ (recomendación fuerte).

- La EGJ-CI (integral contráctil del esfínter esofágico inferior) debe referenciarse a la presión intragástrica y debe expresarse en unidades de $\mathrm{mmHg} \bullet \mathrm{cm}$ (recomendación fuerte). Aunque no cumplió con el nivel de acuerdo, se sugirió que con un valor de $E G J-C I<25 \mathrm{mmHg} \bullet \mathrm{cm}$ se considere la EGJ hipotensa ${ }^{78-86}$.

- La separación LES-CD debería puntuarse como la distancia entre el centro del $C D$ y la señal del LES durante la inspiración, a menos que esté oculta. En este caso la posición del LES se debe puntuar en la espiración (recomendación fuerte).

- El complejo EGJ se debe definir con base en la separación $L E S-C D$ y la localización del RIP (GRADE bajo, recomendación condicional $^{87-93}$.

En cuanto a la morfología de la $E G J$, se reconoció que había tres subtipos: 1) Normal con el CD superpuesto al LES y el RIP en localización proximal al complejo. 2) Separación LES$C D$ con el RIP en localización proximal al $C D$. 3) Separación $L E S-C D$ con el RIP en localización proximal al LES. Sin embargo, no hubo consenso respecto al esquema de clasificación por subtipos (I, II, III, vs A, C, B) y ambas versiones fueron consideradas aceptables (Figura 54$)^{87,92}$. Claramente se necesita más investigación aplicando una metodología estandarizada, previamente especificada. La revisión técnica métricas de la EGJ expone en detalle más recomendaciones respecto a la medición del complejo EGJ en el escenario de hernia hiatal y variabilidad temporal, así como recomendaciones respecto a la medición de la presión intragástrica y el papel de la presión del LES al final de la espiración.

\section{CONCLUSIÓN}

La CCv4.0 es el esquema de clasificación la actualización de las alteraciones de la motilidad esofágica y se deriva de una iniciativa internacional de dos años que contó con la participación de 52 expertos en motilidad esofágica en representación de las sociedades profesionales de los cinco continentes. La Clasificación de Chicago siempre ha sido un proceso dinámico, sujeto a revisión y refinamiento con cada nueva iteración. Honrando este concepto, la CCv4.0 presenta actualizaciones que mejoran la precisión de los diagnósticos de motilidad previamente caracterizados, y elimina diagnósticos infrecuentes o criterios clínicos irrelevantes. Una actualización clave en CCv4.0 es el reconocimiento de que, así como ocurre con otros estudios médicos, los patrones de HRM por sí solos pueden no resultar equivalentes a un diagnóstico conclusivo que explique los síntomas del paciente y guíe el manejo efectivo (patología accionable). Así, la CCv4.0 separa los patrones que sí proporcionan un diagnóstico conclusivo (como acalasia) de otros patrones que sugieren pero no son conclusivos de un diagnóstico, donde la información clínica adicional y las prueba de apoyo pueden confirmar o refutar el diagnóstico en cuestión. En particular, la EGJOO, 
el esófago hipercontráctil y el $D E S$ son patrones manométricos que requieren presencia de disfagia y/o dolor torácico no cardiogénico para ser considerados clínicamente relevantes. Más aún, el diagnóstico conclusivo de EGJOO requiere corroboración con al menos una prueba de apoyo (como TBE, prueba con FLIP).

El rigor metodológico que se ha aplicado al proceso de la CCv4.0 consiste en el uso de métodos de consenso formales y revisión formal del nivel de evidencia cuando han sido aplicables. Adicionalmente, la CCv4.0 recomienda un protocolo de HRM estandarizado para mejorar la consistencia técnica y la exactitud diagnóstica, de manera que las versiones futuras de la Clasificación de Chicago puedan confiar en investigación ulterior con una recolección exhaustiva y uniforme de datos. Finalmente, la CCv4.0 representa las perspectivas respecto a la motilidad de un grupo de trabajo diverso en términos de ubicación geográfica, edad, género, tipo de práctica, años de experiencia, y contribuciones a la investigación en la materia.

La CCv4.0 también resalta las áreas que están listas para futura investigación y clarificación, como se resume más adelante en las revisiones técnicas específicas. Las versiones futuras de la Clasificación de Chicago necesitarán delinear el papel de la topografía por impedancia de la presión de bolo y el flujo del bolo. Se necesitan estudios de resultados adicionales para entender mejor la confiabilidad de las pruebas de deglución de sólidos y de alimentos en la identificación de la $E G J$ anormal y la función peristáltica con relevancia clínica. Con el refinamiento de los criterios diagnósticos de EGJOO en la CCv4.0, será importante entender la evolución natural y el resultado del tratamiento de la EGJOO, con y sin estudios de apoyo. La comprensión de los mecanismos de las alteraciones esofágicas espásticas sigue siendo de gran interés, como lo es la exploración de superposiciones con la disfunción esofágica inducida por opioides ${ }^{46}$. Se requiere trabajo adicional para entender el espectro de las alteraciones hipercontráctiles, explorando si el esófago en martillo neumático representa un subtipo único con significancia clínica. Las versiones futuras de la Clasificación de Chicago podrían proponer criterios manométricos para selección de terapias, tal y como el papel de la miotomía endoscópica peroral para las alteraciones espásticas, o la estratificación del riesgo y la personalización de la funduplicatura para prevenir la disfagia posterior a la funduplicatura.

\section{Financiamiento}

P01 DK092217 (John E. Pandolfino) del Servicio de Salud Pública de los Estados Unidos.

\section{Conflicto de intereses}

Rena Yadlapati: Acuerdo de Consultoría Institucional: Medtronic, Ironwood Pharmaceuticals, Diversatek; Investigación de apoyo: Ironwood Pharmaceuticals; Comité Asesor: Phatom Pharmaceuticals.

Peter J. Kahrilas: Consultor: Ironwood Pharmaceuticals; Comité Asesor: Ironwood Pharmaceuticals; Subvención para investigación: Ironwood Pharmaceuticals; Regalías: Medtronic; Concesión de licencia: Medtronic. 
Mark R. Fox: Conferencista: Medtronic, Diversatek, Laborie; Subvención para investigación: Medtronic, Diversatek, Laborie.

Albert J. Bredenoord: Consultor: Medtronic, Laborie, AstraZeneca, Gossamer, Arena, EsoCap, Calypso, Regeneron, Dr Falk; Conferencista: Medtronic, Laborie, Dr Falk; Comité Asesor: Medtronic, Laborie, AstraZeneca, Gossamer, Arena, EsoCap, Calypso, Regeneron, Dr Falk; Subvenciones para Investigación: Medtronic, Laborie, Nutricia, SST; Opción de compra de acciones: SST.

C. Prakash Gyawali: Consultor: Medtronic, Diversatek, Ironwood Pharmaceuticals, Isothrive, Quintiles.

Sabine Roman: Consultor: Medtronic: Subvenciones para Investigación: Medtronic, Diversatek Healthcare.

Nathalie Rommel: Patente en la tecnología de Manometría de Impedancia Automatizada.

Edoardo Savarino: Consultor: Unifarco; Conferencista: Medtronic, Reckitt Benckiser, Takeda, Abbvie, Novartis, Sandoz, Sofar, Malesci, Janssen, Grifols, Aurora Pharma, Innovamedica, Johnson\&Johnson, SILA, Alfasigma, Shire, EG Stada Group; Comité Asesor: Merck \& Co, Bristol-Myers Squibb, Amgen, Fresenius Kabi; Subvención para investigación: Sofar.

Michael F. Vaezi: Consultor: Diversatek; Phathom; Ironwood; Conferencista: Diversateak; Comité Asesor: Diversatek; Phathom; Ironwood; Subvenciones para Investigación: Diversatek; Phathom; Ironwood; Patente: Mucosal Integrity Testing.
Frank Zerbib: Consultor: Reckitt Benckiser.

Junichi Akiyama: Conferencista: StarMedical, Takeda, AstraZeneca, Otsuka, Daiichi Sankyo.

Serhat Bor: Conferencista: Laborie.

Dustin A. Carlson: Consultor: Medtronic, Conferencista: Medtronic.

Daniel Cisternas: Conferencista: Medtronic.

Enrique Coss-Adame: Consultor: Asofarma de México, Ferrer de México, Siegfried Rhein, Takeda de México, Medtronic de México; Conferencista: Asofarma de México, Ferrer de México, Siegfried Rhein, Takeda de México, Medtronic de México, Grunenthal de México; Comité Asesor: Asofarma de México, Ferrer de México, Siegfried Rhein, Takeda de México, Medtronic de México.

Ronnie Fass: Consultor: Ironwood, Takeda, Daewoong, Medtronic, Phathom; Conferencista: AstraZeneca, Takeda, Diversatek, Eisai; Comité Asesor: Phathom; Subvenciones para Investigación: Ironwood, Salix; Regalías: UpToDate.

Philip Katz: Consultor: Medtronic; Subvención para Investigación: Diversatek.

David A. Katzka: Consultor: Shire.

Abraham Khan: Consultor: Medtronic; Conferencista: Medtronic.

Daniel Pohl: Consultor: Medtronic, Sanofi; Conferencista: Permamed, Alfa Sigma, Schwabe Pharma, Takeda; Comité Asesor: Sanofi, Schwabe Pharma. 
Jordi Serra: Consultor: Reckit Benckiser; Conferencista: Norgine, Allergan, Cassen Recordati, AB biotics; Comité Asesor: Almirall; Subvenciones para Investigación: Bayer, Salvat Biotech, Zespri.

Rami Sweis: Conferencista: Medtronic, Covidian, Given, Falk Pharma, Ethicon; Comité Asesor: Falk Pharma, Ethicon.

Jan Tack: Subvención para Investigación: Sofar Pharmaceuticals.

Radu Tutuian: Consultor: Laborie MMS; Conferencista: Laborie MMS; Regalías: UpToDate.

Marcelo F. Vela: Consultor: Medtronic.

Reuben Wong: Consultor: MMS Laborie, Conferencista: MMS Laborie.

John E. Pandolfino: Consultor: Medtronic, Ironwood Pharmaceuticals, Diversatek; Apoyo en Investigación: Ironwood Pharmaceuticals, Takeda; Comité Asesor: Medtronic, Diversatek; Opción de compra de Acciones: Crospon Inc.

Ningún conflicto de intereses para declarar: Arash Babaei, Ravinder K. Mittal, Daniel Sifrim, Andre Smout, Shobna Bhatia, Joan Chen, Charles Cock, Nicola de Bortoli, Claudia Defilippi, Uday C. Ghoshal, Sutep Gonlachanvit, Albis Hani, Geoffrey S. Hebbard, Kee Wook Jung, Geoffrey P. Kohn, Adriana Lazarescu, Johannes Lenglinger, Sumeet K. Mittal, Taher Omari, Moo In Park, Roberto Penagini, Joel E. Richter, Roger P. Tatum, Justin $\mathrm{Wu}$, Yinglian Xiao.

\section{RESPONSABILIDADES ÉTICAS}

Protección de personas y animales. Los autores declaran que para esta investigación no se han realizado experimentos en seres humanos ni en animales.

Confidencialidad de los datos. Los autores declaran que han seguido los protocolos de su centro de trabajo sobre la publicación de datos de pacientes.

\section{Derecho a la privacidad y consentimiento} informado. Los autores han obtenido el consentimiento informado de los pacientes y/o sujetos referidos en el artículo. Este documento obra en poder del autor de correspondencia.

\section{Material suplementario}

El material suplementario se encuentra a disposición de los lectores en: https://onlinelibrary.wiley.com/doi/suppl/10.1111/nmo.14058.

\section{BIBLIOGRAFÍA}

1. Yadlapati R, Kahrilas PJ, Fox MR, et al. Esophageal motility disorders on high-resolution manometry: Chicago classification version $4.0^{\circ}$. Neurogastroenterol Motil. 2021 Jan;33(1):e14058. doi: 10.1111/nmo.14058. PMID: 33373111; PMCID: PMC8034247.

2. Kahrilas PJ, Bredenoord AJ, Fox M, et al. The Chicago classification of esophageal motility disorders, v3.0. Neurogastroenterol Motil. 2015;27:160-174.

3. Pandolfino JE, Fox MR, Bredenoord AJ, et al. High-resolution manometry in clinical practice: utilizing pressure topography to classify oesophageal motility abnormalities. Neurogastroenterol Motil. 2009;21:796-806.

4. Bredenoord AJ, Fox M, Kahrilas PJ, et al. Chicago classification criteria of esophageal motility disorders defined in high resolution esophageal pressure topography. Neurogastroenterol Motil. 2012;24(Suppl 1):57-65.

5. Yadlapati R, Gawron AJ, Keswani RN, et al. Identification of quality measures for performance of and interpretation of data from esophageal manometry. Clin Gastroenterol Hepatol. 2016;14:526-534.

6. Yadlapati R, Vaezi MF, Vela MF, et al. Management options for patients with GERD and persistent symptoms on proton pump inhibitors: recommendations from an expert panel. Am J Gastroenterol. 2018;113:980-986. 
7. Balshem H, Helfand M, Schünemann HJ, et al. GRADE guidelines: 3. Rating the quality of evidence. J Clin Epidemiol. 2011;64:401-406.

8. Shaker A, Stoikes N, Drapekin J, et al. Multiple rapid swallow responses during esophageal high-resolution manometry reflect esophageal body peristaltic reserve. Am J Gastroenterol. 2013;108:1706-1712.

9. Mauro A, Savarino E, De Bortoli N, et al. Optimal number of multiple rapid swallows needed during high-resolution esophageal manometry for accurate prediction of contraction reserve. Neurogastroenterol Motil. 2018;30:e13253.

10. Woodland P, Gabieta-Sonmez S, Arguero J, et al. $200 \mathrm{~mL}$ rapid drink challenge during high-resolution manometry best predicts objective esophagogastric junction obstruction and correlates with symptom severity. J Neurogastroenterol Motil. 2018;24:410-414.

11. Ang D, Misselwitz B, Hollenstein M, et al. Diagnostic yield of high-resolution manometry with a solid test meal for clinically relevant, symptomatic oesophageal motility disorders: serial diagnostic study. Lancet Gastroenterol Hepatol. 2017;2:654-661.

12. Babaei A, Shad S, Szabo A, et al. Pharmacologic interrogation of patients with esophagogastric junction outflow obstruction using amyl nitrite. Neurogastroenterol Motil. 2019;31:e13668.

13. Triggs JR, Carlson DA, Beveridge C, et al. Functional luminal imaging probe panometry identifies achalasia-type esophagogastric junction outflow obstruction. Clin Gastroenterol Hepatol. 2020;18:2209-2217.

14. Clayton SB, Patel R, Richter JE. Functional and anatomic esophagogastic junction outflow obstruction: manometry, timed barium esophagram findings, and treatment outcomes. Clin Gastroenterol Hepatol. 2016; 14:907-911

15. Herregods TVK, Roman S, Kahrilas PJ, et al. Normative values in esophageal high-resolution manometry. Neurogastroenterol Motil. 2015;27:175-187.

16. Kuribayashi S, Iwakiri K, Kawada A, et al. Variant parameter values-as defined by the Chicago criteria-produced by ManoScan and a new system with Unisensor catheter. Neurogastroenterol Motil. 2015;27:188-194.

17. Sifrim D, Roman S, Savarino E, et al. Normal values and regional differences in oesophageal impedance-pH metrics: a consensus analysis of impedance-pH studies from around the world. Gut. 2020. https://doi. org/10.1136/gutjnl-2020-322627. [Epub ahead of print].

18. Rengarajan A, Rogers BD, Wong Z, et al. High-resolution manometry thresholds and motor patterns among asymptomatic individuals. Clin Gastroenterol Hepatol. 2020. https://doi.org/10.1016/j. cgh.2020.10.052. [Epub ahead of print].

19. Triggs JR, Carlson DA, Beveridge C, et al. Upright integrated relaxation pressure facilitates characterization of esophagogastric junction outflow obstruction. Clin Gastroenterol Hepatol. 2019;17:2218-2226.

20. Pandolfino JE, Roman S, Carlson D, et al. Distal esophageal spasm in highresolution esophageal pressure topography: defining clinical phenotypes. Gastroenterology. 2011;141:469-475.

21. Roman S, Kahrilas PJ, Kia L, et al. Effects of large hiatal hernias on esophageal peristalsis. Arch Surg. 2012;147:352-357.

22. Pandolfino JE, Kwiatek MA, Nealis T, et al. Achalasia: a new clinically relevant classification by high-resolution manometry. Gastroenterology. 2008;135:1526-1533.

23. Biasutto D, Mion F, Garros A, et al. Rapid drink challenge test during esophageal high resolution manometry in patients with esophago-gastric junction outflow obstruction. Neurogastroenterol Motil. 2018;30:e13293.

24. Song BG, Min YW, Lee H, et al. Clinicomanometric factors associated with clinically relevant esophagogastric junction outflow obstruction from the Sandhill high-resolution manometry system. Neurogastroenterol Motil. 2018;30(3):e13221.

25. lvevi A, Mauro A, Pugliese D, et al. Usefulness of low- and high-volume multiple rapid swallowing during high-resolution manometry. Dig Liver Dis. 2015;47:103-107.

26. Martinucci I, Savarino EV, Pandolfino JE, et al. Vigor of peristalsis during multiple rapid swallows is inversely correlated with acid exposure time in patients with NERD. Neurogastroenterol Motil. 2016;28:243-250.
27. Krause AJ, Su H, Triggs JR, et al. Multiple rapid swallows and rapid drink challenge in patients with esophagogastric junction outflow obstruction on high-resolution manometry. Neurogastroenterol Motil. 2020:e14000. https://doi.org/10.1111/nmo.14000. [Epub ahead of print].

28. Ang D, Hollenstein M, Misselwitz B, et al. Rapid drink challenge in highresolution manometry: an adjunctive test for detection of esophageal motility disorders. Neurogastroenterol Motil. 2017;29(1):e12902.

29. Marin I, Serra J. Patterns of esophageal pressure responses to a rapid drink challenge test in patients with esophageal motility disorders. Neurogastroenterol Motil. 2016;28:543-553.

30. Sanagapalli S, McGuire J, Leong RW, et al. The clinical relevance of manometric esophagogastric junction outflow obstruction can be determined using rapid drink challenge and solid swallows. Am J Gastroenterol. 2020. https://doi.org/10.14309/ajg.0000000000 000988. [Epub ahead of print].

31. Hollenstein M, Thwaites P, Bütikofer S, et al. Pharyngeal swallowing and oesophageal motility during a solid meal test: a prospective study in healthy volunteers and patients with major motility disorders. Lancet Gastroenterol Hepatol. 2017;2:644-653.

32. Babaei A, Shad S, Massey BT. Diagnostic differences in the pharmacologic response to cholecystokinin and amyl nitrite in patients with absent contractility vs type I achalasia. Neurogastroenterol Motil. 2020;32:e13857.

33. Babaei A, Shad S, Massey BT. Motility patterns following esophageal pharmacologic provocation with amyl nitrite or cholecystokinin during highresolution manometry distinguish idiopathic vs opioid-induced type 3 achalasia. Clin Gastroenterol Hepatol. 2020;18(4):813-821.

34. Andolfi C, Fisichella PM. Meta-analysis of clinical outcome after treatment for achalasia based on manometric subtypes. Br J Surg. 2019;106:332-341.

35. Lee JY, Kim N, Kim SE, et al. Clinical characteristics and treatment outcomes of 3 subtypes of achalasia according to the Chicago classification in a tertiary institute in Korea. J Neurogastroenterol Motil. 2013;19:485-494.

36. Min M, Peng LH, Yang YS, et al. Characteristics of achalasia subtypes in untreated Chinese patients: a high-resolution manometry study. J Dig Dis. 2012;13:504-509.

37. Rohof WO, Salvador R, Annese V, et al. Outcomes of treatment for achalasia depend on manometric subtype. Gastroenterology. 2013;144(4):718-725.

38. Blonski W, Kumar A, Feldman J, et al. Timed barium swallow: diagnostic role and predictive value in untreated achalasia, esophagogastric junction outflow obstruction, and non-achalasia dysphagia. Am J Gastroenterol. 2018;113:196-203.

39. Clayton SB, Shin CM, Ewing A, et al. Pneumatic dilation improves esophageal emptying and symptoms in patients with idiopathic esophago-gastric junction outflow obstruction. Neurogastroenterol Motil. 2019;31:e13522.

40. Okeke FC, Raja S, Lynch KL, et al. What is the clinical significance of esophagogastric junction outflow obstruction? Evaluation of 60 patients at a tertiary referral center. Neurogastroenterol Motil. 2017;29(6):e13061.

41. Carlson DA, Kahrilas PJ, Lin Z, et al. Evaluation of esophageal motility utilizing the functional lumen imaging probe. Am J Gastroenterol. 2016;111:1726-1735.

42. Kim E, Yoo IK, Yon DK, et al. Characteristics of a subset of achalasia with normal integrated relaxation pressure. J Neurogastroenterol Motil. 2020;26:274-280.

43. Ponds FA, Bredenoord AJ, Kessing BF, et al. Esophagogastric junction distensibility identifies achalasia subgroup with manometrically normal esophagogastric junction relaxation. Neurogastroenterol Motil. 2017;29(1):e12908

44. Sanagapalli S, Roman S, Hastier A, et al. Achalasia diagnosed despite normal integrated relaxation pressure responds favorably to therapy. Neurogastroenterol Motil. 2019;31:e13586.

45. Babaei A, Szabo A, Shad S, et al. Chronic daily opioid exposure is associated with dysphagia, esophageal outflow obstruction, and disordered peristalsis. Neurogastroenterol Motil. 2019;31:e13601. 
46. Ratuapli SK, Crowell MD, DiBaise JK, et al. Opioid-induced esophageal dysfunction (OIED) in patients on chronic opioids. Am J Gastroenterol. 2015;110:979-984.

47. Misselwitz B, Hollenstein M, Bütikofer S, et al. Prospective serial diagnostic study: the effects of position and provocative tests on the diagnosis of oesophageal motility disorders by high-resolution manometry. Aliment Pharmacol Ther. 2020;51:706-718.

48. Beveridge CA, Falk GW, Ahuja NK, et al. Low yield of cross-sectional imaging in patients with esophagogastric junction outflow obstruction. Clin Gastroenterol Hepatol. 2020;18:1643-1644

49. DeLay K, Austin GL, Menard-Katcher P. Anatomic abnormalities are common potential explanations of manometric esophagogastric junction outflow obstruction. Neurogastroenterol Motil. 2016;28:1166-1171.

50. Liu A, Woo M, Nasser Y, et al. Esophagogastric junction outflow obstruction on manometry: outcomes and lack of benefit from CT and EUS. Neurogastroenterol Motil. 2019;31:e13712.

51. Pérez-Fernández M-T, Santander C, Marinero A, et al. Characterization and follow-up of esophagogastric junction outflow obstruction detected by high resolution manometry. Neurogastroenterol Motil. 2016;28:116-126.

52. van Hoeij FB, Smout AJ, Bredenoord AJ. Characterization of idiopathic esophagogastric junction outflow obstruction. Neurogastroenterol Motil. 2015;27:1310-1316.

53. Wang YT, Tai LF, Yazaki E, et al. Investigation of dysphagia after antireflux surgery by high-resolution manometry: impact of multiple water swallows and a solid test meal on diagnosis, management, and clinical outcome. Clin Gastroenterol Hepatol. 2015;13:1575-1583.

54. Hoscheit M, Gabbard S. Elevated intrabolus pressure predicts abnormal timed barium esophagram in esophagogastric junction outflow obstruction. J Neurogastroenterol Motil. 2019;25:521-524.

55. Quader F, Reddy C, Patel A, et al. Elevated intrabolus pressure identifies obstructive processes when integrated relaxation pressure is normal on esophageal high-resolution manometry. Am J Physiol Gastrointest Liver Physiol. 2017;313:G73-G79.

56. Singendonk MJ, Lin Z, Scheerens C, et al. High-resolution impedance manometry parameters in the evaluation of esophageal function of non-obstructive dysphagia patients. Neurogastroenterol Motil. 2019;31:e13505.

57. Schupack D, Katzka DA, Geno DM, et al. The clinical significance of esophagogastric junction outflow obstruction and hypercontractile esophagus in high resolution esophageal manometry. Neurogastroenterol Motil. 2017;29:1-9.

58. Song BG, Min YW, Lee H, et al. Combined multichannel intraluminal impedance and high-resolution manometry improves detection of clinically relevant esophagogastric junction outflow obstruction. J Neurogastroenterol Motil. 2019;25:75-81.

59. Babaei A, Lin EC, Szabo A, et al. Determinants of pressure drift in Manoscan() esophageal high-resolution manometry system. Neurogastroenterol Motil. 2015;27:277-284.

60. Babaei A, Szabo A, Yorio SD, et al. Pressure exposure and catheter impingement affect the recorded pressure in the Manoscan 360 system. Neurogastroenterol Motil. 2018;30(8):e13329.

61. Zerbib F, Marin I, Cisternas D, et al. Ineffective esophageal motility and bolus clearance. A study with combined high-resolution manometry and impedance in asymptomatic controls and patients. Neurogastroenterol Motil. 2020;32(9):e13876.

62. Rogers BD, Rengarajan A, Mauro A, et al. Fragmented and failed swallows on esophageal high-resolution manometry associate with abnormal reflux burden better than weak swallows. Neurogastroenterol Motil. 2020;32:e13736

63. Herregods TVK, Smout AJPM, Ooi JLS, et al. Jackhammer esophagus: observations on a European cohort. Neurogastroenterol Motil. 2017; 29(4):e12975.

64. Kahn A, Al-Qaisi MT, Obeid RA, et al. Clinical features and longterm outcomes of lower esophageal sphincter-dependent and lower esophageal sphincterindependentjackhammer esophagus. Neurogastroenterol Motil. 2019;31:e13507.
65. Roman S, Pandolfino JE, Chen J, et al. Phenotypes and clinical context of hypercontractility in high-resolution esophageal pressure topography (EPT). Am J Gastroenterol. 2012;107:37-45.

66. Al-Qaisi MT, Siddiki HA, Crowell MD, et al. The clinical significance of hypercontractile peristalsis: comparison of high-resolution manometric features, demographics, symptom presentation, and response to therapy in patients with Jackhammer esophagus versus Nutcracker esophagus. Dis Esophagus. 2017;30:1-7.

67. Philonenko S, Roman S, Zerbib F, et al. Jackhammer esophagus: clinical presentation, manometric diagnosis, and therapeutic results-Results from a multicenter French cohort. Neurogastroenterol Motil. 2020;32(11):e13918.

68. Xiao Y, Carlson DA, Lin Z, et al. Jackhammer esophagus: assessing the balance between prepeak and postpeak contractile integral. Neurogastroenterol Motil. 2018;30:e13262.

69. Xiao Y, Carlson DA, Lin Z, et al. Chaotic peak propagation in patients with Jackhammer esophagus. Neurogastroenterol Motil. 2020;32:e13725.

70. Bulsiewicz WJ, Kahrilas PJ, Kwiatek MA, et al. Esophageal pressure topography criteria indicative of incomplete bolus clearance: a study using highresolution impedance manometry. Am J Gastroenterol. 2009;104:2721-2728.

71. Jain A, Baker JR, Chen JW. In ineffective esophageal motility, failed swallows are more functionally relevant than weak swallows. Neurogastroenterol Motil. 2018;30:e13297

72. Roman S, Lin Z, Kwiatek MA, et al. Weak peristalsis in esophageal pressure topography: classification and association with dysphagia. Am J Gastroenterol. 2011;106:349-356.

73. Rengarajan A, Bolkhir A, Gor P, et al. Esophagogastric junction and esophageal body contraction metrics on high-resolution manometry predict esophageal acid burden. Neurogastroenterol Motil. 2018;30:e13267.

74. Chugh P, Collazo T, Dworkin B, et al. Ineffective esophageal motility is associated with impaired bolus clearance but does not correlate with severity of dysphagia. Dig Dis Sci. 2019;64:811-814.

75. Lin Z, Nicodème F, Lin C-Y, et al. Parameters for quantifying bolus retention with high-resolution impedance manometry. Neurogastroenterol Motil. 2014;26:929-936.

76. Wu J-F, Tsai I-J, Tong T-W, et al. Pressure-impedance analysis: assist the diagnosis and classification of ineffective esophageal motility disorder. J Gastroenterol Hepatol. 2020;35:1317-1324.

77. Quader F, Rogers B, Sievers T, et al. Contraction reserve with ineffective esophageal motility on esophageal high-resolution manometry is associated with lower acid exposure times compared with absent contraction reserve. Am J Gastroenterol. 2020;115(12):1981- 1988. https://doi. org/10.14309/ajg.0000000000000811

78. Gor P, Li Y, Munigala S, et al. Interrogation of esophagogastric junction barrier function using the esophagogastric junction contractile integral: an observational cohort study. Dis Esophagus. 2016;29:820-828.

79. Ham H, Cho YK, Lee HH, et al. Esophagogastric junction contractile integral and morphology: two high-resolution manometry metrics of the antireflux barrier. J Gastroenterol Hepatol. 2017;32:1443-1449.

80. Jasper D, Freitas-Queiroz N, Hollenstein M, et al. Prolonged measurement improves the assessment of the barrier function of the esophago-gastric junction by high-resolution manometry. Neurogastroenterol Motil. 2017;29(2). https://doi.org/10.1111/nmo.12925

81. Nicodème F, Pipa-Muniz M, Khanna K, et al. Quantifying esophagogastric junction contractility with a novel HRM topographic metric, the EGJ-Contractile Integral: normative values and preliminary evaluation in PPI non-responders. Neurogastroenterol Motil. 2014;26:353360.

82. Rengarajan A, Gyawali CP. High-resolution manometry can characterize esophagogastric junction morphology and predict esophageal reflux burden. J Clin Gastroenterol. 2020;54:22-27.

83. Tolone S, De Bortoli N, Marabotto E, et al. Esophagogastric junction contractility for clinical assessment in patients with GERD: a real added value? Neurogastroenterol Motil. 2015;27:1423-1431. 
84. Wang D, Patel A, Mello M, et al. Esophagogastric junction contractile integral (EGJ-CI) quantifies changes in EGJ barrier function with surgical intervention. Neurogastroenterol Motil. 2016;28:639-646.

85. . Xie C, Wang J, Li Y, et al. Esophagogastric junction contractility integral reflect the anti-reflux barrier dysfunction in patients YADLAPATI et al. | 21 of 21 with gastroesophageal reflux disease. J Neurogastroenterol Motil. 2017;23:27-33

86. Rogers BD, Rengarajan A, Abrahao L, et al. Esophagogastric junction morphology and contractile integral on high-resolution manometry in asymptomatic healthy volunteers: an international multicenter study. Neurogastroenterol Motil. 2020:e14009. https://doi.org/10.1111/nmo.14009. [Epub ahead of print].

87. Akimoto S, Singhal S, Masuda T, et al. Classification for esophagogastric junction (EGJ) complex based on physiology. Dis Esophagus. 2017;30:1-6.

88. Akimoto S, Singhal S, Masuda T, et al. Esophagogastric junction morphology and distal esophageal acid exposure. Dig Dis Sci. 2016;61:3537-3544.
89. Mittal RK, Zifan A, Kumar D, et al. Functional morphology of the lower esophageal sphincter and crural diaphragm determined by three-dimensional high-resolution esophago-gastric junction pressure profile and CT imaging. Am J Physiol Gastrointest Liver Physiol. 2017;313:G212-G219.

90. Nicodème F, Lin Z, Pandolfino JE, et al. Esophagogastric Junction pressure morphology: comparison between a station pull-through and real-time 3D-HRM representation. Neurogastroenterol Motil. 2013;25:e591-e598.

91. Niebisch S, Wilshire CL, Peters JH. Systematic analysis of esophageal pressure topography in high-resolution manometry of 68 normal volunteers. Dis Esophagus. 2013;26:651-660.

92. Pandolfino JE, Kim H, Ghosh SK, et al. High-resolution manometry of the EGJ: an analysis of crural diaphragm function in GERD. Am J Gastroenterol. 2007;102:1056-1063. 92.

93. Tolone S, de Cassan C, de Bortoli N, et al. Esophagogastric junction morphology is associated with a positive impedance-pH monitoring in patients with GERD. Neurogastroenterol Motil. 2015;27:1175-1182. 